Draft VERSION SEPTEMBER 19, 2018

Preprint typeset using LATEX style AASTeX6 v. 1.0

\title{
TRACING THE MAGNETIC FIELD OF IRDC G028.23-00.19 USING NIR POLARIMETRY
}

\author{
Sadia Hoq ${ }^{1}$, D. P. Clemensi ${ }^{1}$, Andrés E. Guzmán ${ }^{2}$, and Lauren R. Cashman ${ }^{1}$ \\ ${ }^{1}$ Institute for Astrophysical Research, 725 Commonwealth Ave, Boston University, Boston, MA 02215; shoq@bu.edu, clemens@bu.edu, \\ lcashman@bu.edu \\ ${ }^{2}$ Departamento de Astronomía, Universidad de Chile, Camino el Observatorio 1515, Las Condes, Santiago, Chile, aguzman@das.uchile.cl
}

\begin{abstract}
The importance of the magnetic (B) field in the formation of Infrared Dark Clouds (IRDCs) and massive stars is an ongoing topic of investigation. We studied the plane-of-sky B-field for one IRDC, G028.23-00.19, to understand the interaction between the field and the cloud. We used near-IR background starlight polarimetry to probe the B-field, and performed several observational tests to assess the field importance. The polarimetric data, taken with the Mimir instrument, consisted of $H$-band and $K$-band observations, totaling 17,160 stellar measurements. We traced the planeof-sky B-field morphology with respect to the sky-projected cloud elongation. We also found the relationship between the estimated B-field strength and gas volume density, and computed estimates of the normalized Mass-to-Magnetic Flux ratio. The B-field orientation with respect to the cloud did not show a preferred alignment, but did exhibit a large-scale pattern. The plane-of-sky B-field strengths ranged from 10-165 $\mu \mathrm{G}$, and the B-field strength dependence on density followed a power law with an index consistent with 2/3. The Mass-to-Magnetic Flux ratio also increased as a function of density. The relative orientations and relationship between B-field and density imply that the B-field was not dynamically important in the formation of the IRDC. The increase in Mass-to-Flux ratio as a function of density, though, indicates a dynamically important B-field. Therefore, it is unclear whether the B-field influenced the formation of G28.23. However, it is likely that the presence of the IRDC changed the local B-field morphology .

Keywords: ISM:clouds-ISM:magnetic fields-ISM:dust, extinction—stars:formation—Individual Object:G28.23 - techniques:polarimetric
\end{abstract}

\section{INTRODUCTION}

Infrared Dark Clouds (IRDCs) are dense $\left(\mathrm{H}_{2}\right.$ column densities $\sim 10^{22}-10^{23} \mathrm{~cm}^{-2}$ ) filamentary structures that host high-mass star formation (Rathborne et al. 2006). As such, these clouds play important roles in the evolution of the Galaxy. However, much is still not known about IRDC formation and evolution. One open problem is the unknown role of magnetic (B) fields in the formation of IRDCs and any potential star formation they host.

While many studies have probed the B-fields of molecular clouds using techniques such as the Zeeman effect, background starlight polarimetry, and dust emission polarimetry (e.g., Troland \& Crutcher 2008; Chapman et al. 2011; Dotson et al. 2010), studies of the Bfields of IRDCs have mainly focused on the small size scales of cores and clumps (e.g., Cortes et al. 2008; Tang et al. 2009; Girart et al. 2009; Sridharan et al. 2014; Zhang et al. 2014). Several studies have probed the interstellar medium (ISM) between clouds (Heiles 2000; Li et al. 2009), but few have probed the cloud scales (10s of pc) to study the interaction between the B-field and an IRDC as a whole (e.g., Sugitani et al. 2011; Pillai et al. 2015).

To test whether B-fields play a dominant role in IRDC and massive star formation (e.g., Nakamura \& Li 2008), or are themselves influenced by more dominant forces (e.g., Padoan \& Nordlund 1999), such as turbulence or gravity, B-field properties must be observationally related to other physical cloud properties.

Many models and simulations have studied the formation of filamentary molecular clouds and the forces that control their formation (e.g., Nakajima \& Hanawa 1996; Ostriker et al. 2001; Padoan \& Nordlund 2002; Hennebelle 2013; Van Loo et al. 2014; Li et al. 2015a). Several of these studies (e.g., Ostriker et al. 2001; Van Loo et al. 2014; Li et al. 2015a) considered the role of B-fields in cloud formation to predict the observational signatures of weak and strong B-fields. One of the most straightforward signatures is the relative orientation of the B-field with respect to the filament orientations. For example, the preferential direction of B-fields relative 
to cloud orientation can reveal whether material flowed along field lines to create clouds. If the field is more likely found to be either perpendicular or parallel to the cloud orientation, then the B-field very likely played a role in the cloud's formation (Li et al. 2009).

Other observational tests rely on the relative strength of the B-field. The relative strength of the gravitational potential compared to the local B-field flux ('Mass-toFlux' ratio, $\mathrm{M} / \Phi_{B}$, see review by Crutcher 2012) of a clump reveals the importance of the B-field compared to gravity. Additionally, if the power-law dependence of the B-field strength on cloud gas volume density is shallower than $2 / 3$, the field likely influenced the flow of material during cloud formation (Li et al. 2015a).

Near-Infrared (NIR) background starlight polarimetry provides a way to probe the B-field on scales of $\sim 1$ to tens of pc at the distances to most IRDCs. The polarization signal is caused by aspherical dust grains spinning with their long axes aligned mostly perpendicular to the intervening B-field (Lazarian \& Hoang 2007). The linear polarization signal imparted on background starlight by the asymmetric dichroic extinction of the dust grains follows the orientation of the B-field in the plane of the sky. Therefore, the orientations of the NIR polarizations trace the plane-of-sky B-field. NIR polarimetry can reveal the plane-of-sky B-field morphology over large fields of view and wide ranges of column and volume densities (e.g., Clemens et al. 2012b). In addition, the plane-ofsky B-field strength can often be inferred by using the Chandrasekhar-Fermi Method (Chandrasekhar \& Fermi 1953, hereafter CF Method), which combines the polarization measurements with complementary cloud density and gas velocity information.

\subsection{IRDC G28.23}

In this study, we evaluated the plane-of-sky B-field toward IRDC G028.23-00.19 (hereafter referred to as G28.23) ( $l=28.23, b=-0.19$; Rathborne et al. 2006), using NIR polarimetric observations, to determine the role of the B-field in the formation of the IRDC. Figure 1 shows a 3-color GLIMPSE and MIPSGAL (Benjamin et al. 2003; Carey et al. 2009) image of G28.23. Both GLIMPSE (Galactic Legacy Infrared Mid-Plane Survey Extraordinaire) and MIPSGAL (MIPS Galactic Plane Survey) are infrared surveys of the Galaxy conducted with the Spitzer Space Telescope. GLIMPSE observations were taken in four bands: $3.6,4.5,5.8$, and $8 \mu \mathrm{m}$, and MIPSGAL observations were taken in 24 and 70 $\mu \mathrm{m}$. The IRDC can be seen as the dark extinction feature against the bright background emission ${ }^{1}$ Lo-

\footnotetext{
1 The bright IR point source at the top left of the cloud is an unassociated OH/IR foreground star, at a LSR radial velocity of
}

cated at a distance of $5.1 \mathrm{kpc}, \mathrm{G} 28.23$ is a dense, quiescent IRDC that hosts one of the most massive quiescent cores found in an IRDC, and is a likely candidate to host future high-mass star formation. Because of its quiescent nature, G28.23 is an ideal laboratory in which to study the interaction between an IRDC and the surrounding B-field. With no signs of active star formation, G28.23 provides an opportunity to study the interaction between an IRDC and the surrounding B-field before any potential disruption by active star formation.

\subsection{Methodology}

We pursued answers to four questions regarding the plane-of-sky B-field, as revealed by deep NIR observations, to ascertain the field's importance in the formation of G28.23.

1. Do polarization percentages, as probed by NIR background starlight polarimetry, increase as a function of extinction? If the polarization percentages do not increase with extinction, then it is possible that the NIR polarization measurements only probe the skin of the cloud (Arce et al. 1998) and do not reveal the B-field properties deeper into the cloud. Depolarization along the line of sight, where different layers of material exhibit different polarization orientations that cancel when summed, can also cause this effect. If the polarization increases with extinction, then this is evidence that the material in G28.23 positively increases the measured polarization percentage. Therefore, the B-field can be probed by NIR polarization of background stars.

2. Is the plane-of-sky B-field preferentially aligned with the major axis orientation of G28.23? Because the angle of the cloud major axis orientation with respect to plane of the sky is not known, we will use the cloud elongation as a proxy for the cloud's major axis. If the B-field is perpendicular or parallel to the cloud elongation, then the field likely played a role in the cloud formation. Otherwise, if the field is randomly oriented with respect to the cloud, the field likely did not strongly influence cloud formation.

3. What is the power-law dependence between B-field strength and cloud volume density? A weak B-field frozen into isotropically collapsing material would follow a power law of $2 / 3$ (Crutcher et al. 2010), whereas a strong field would follow a power law shallower than $2 / 3$.

4. How does $M / \Phi_{B}$ vary across the cloud? While NIR polarimetry will not probe the densest cloud interiors, it will probe the B-field in the outer to middle cloud regions. The $\mathrm{M} / \Phi_{B}$ estimates will reveal the relative

$\sim 52 \mathrm{~km} \mathrm{~s}^{-1}$ (Bowers \& Knapp 1989), which is different from the $80 \mathrm{~km} \mathrm{~s}^{-1}$ velocity of G28.23 (Sanhueza et al. 2013). 


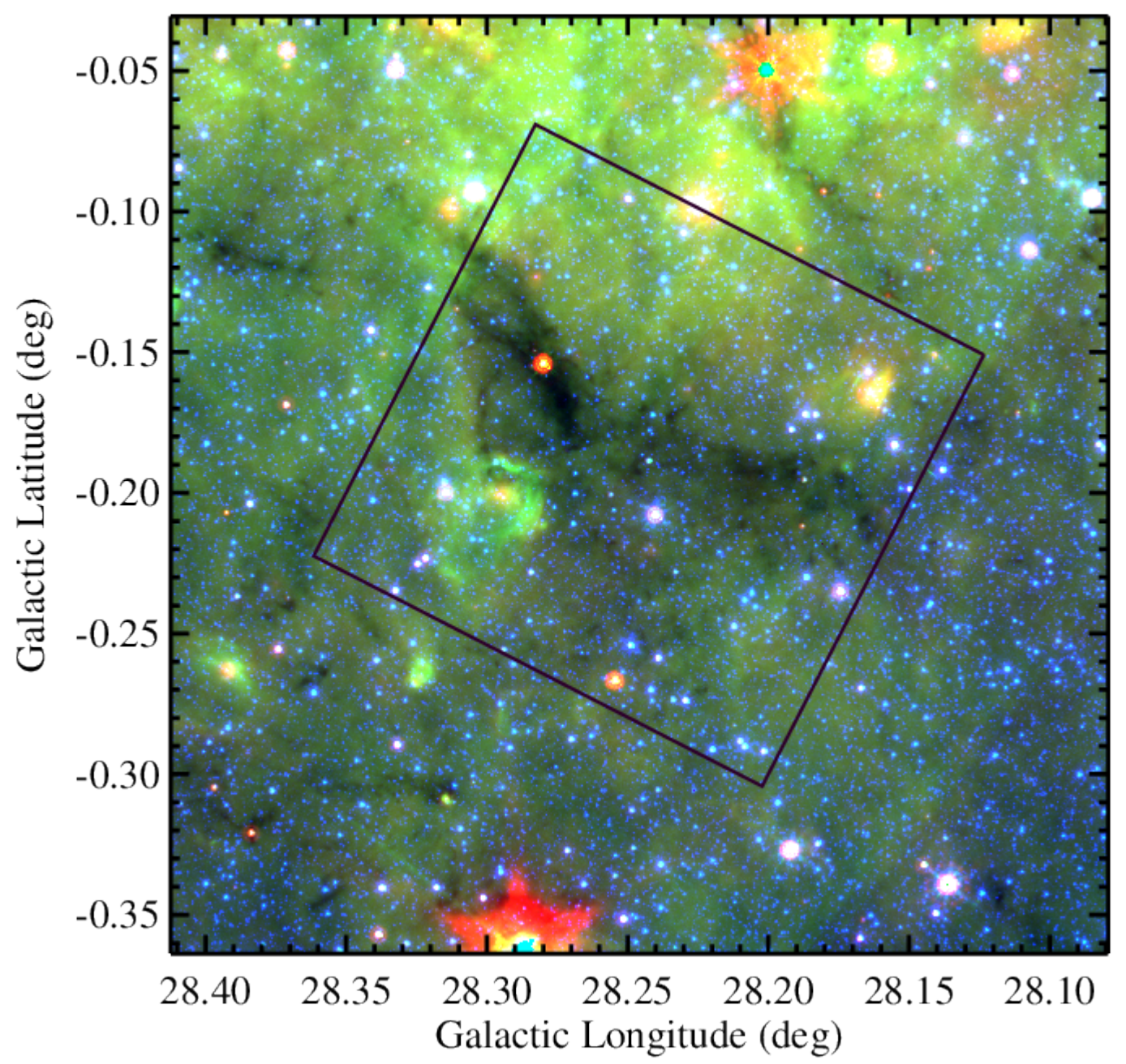

Figure 1. Three-color Spitzer GLIMPSE (Benjamin et al. 2003) and MIPSGAL (Carey et al. 2009) (blue: 3.6, green: 8, red: $24 \mu \mathrm{m}$ ) image of G28.23. A black box representing the field of view of the $K$-band polarimetric observations (approximately $10 \times 10$ armin) is overlaid. The bright source at $l=28.275, b=-0^{\circ} .15$ is an unrelated foreground star.

changes of the B-field strength with respect to gravity in the outer layers of G28.23, especially in the density ranges where ambipolar diffusion is predicted to operate (e.g., Mouschovias 1979).

Using a combination of new and archival NIR polarimetry, archival submm and far-IR (FIR) dust emission, and published molecular line data, we studied the magnetic and physical properties of G28.23. The paper is organized as follows. Section 2 describes the observations and archival data products used. Section 3 outlines the data analysis and results. Section 4 discusses the implications of the results on the importance of the B-field, and Section 5 summarizes the study.

\subsection{NIR Polarimetry}

NIR polarimetric observations in both $H$-band (1.6 $\mu \mathrm{m})$ and $K$-band $(2.2 \mu \mathrm{m})$ of G28.23-00.19 were obtained using the Mimir instrument (Clemens et al. 2007) on the $1.8 \mathrm{~m}$ Perkins Telescope in Flagstaff, AZ. The instrument field of view (FOV) was 10x10 arcmin with a plate scale of 0.58 arcsec per pixel. Mimir used a compound half-wave plate (HWP) in conjunction with a fixed, cold wire-grid. The $H$-band data were taken from Data Release 2 of the Galactic Plane Infrared Polarization Survey (GPIPS, Clemens et al. 2012b) ${ }^{2}$. GPIPS spanned 76 sq. degrees of the inner Galaxy, from 18-

\footnotetext{
2 http://gpips0.bu.edu/Data_Release/
}

\section{OBSERVATIONS}


$56^{\circ}$ in Galactic longitude and $\pm 1^{\circ}$ in latitude, which fully covered the location and extent of G28.23. The survey region consists of 3,237 individual pointings, each covering a 10x10 arcmin area. Each GPIPS observation consisted of 96 images (one each at 16 unique HWP positions at six dither positions on the sky) with an exposure time of 2.5 seconds. The total integration time of each observation was $\sim 4$ minutes. Sky conditions were clear, and each GPIPS field was required to meet a $2 \operatorname{arcsec}$ seeing criterion.

The $H$-band data for a $20 \times 20$ arcmin region (the region shown in Figure 1) covering the IRDC, as well as its environment, were extracted from the GPIPS database. This region is aligned in Galactic coordinates, with center $(l, b)=(28.244,-0.200)$.

Targeted $K$-band observations, covering about $10.4 \times 10.8$ arcmin, centered on coordinates $l=28.247$ and $b=-0$. 19 , and aligned in R.A. and decl., were obtained over five nights in 2013 September and 2014 June. These consisted of 14 separate observations, each with 96 individual 15 second exposures. The total integration time was 5.6 hours. These deep $K$-band observations probed the more extincted regions, while the $H$-band data covered a larger FOV. The $K$-band sky coverage is outlined as the black box in Figure 1. Hereafter, references to the $10 \times 10$ arcmin FOV centered on the cloud refer to this $10.4 \times 10.8$ arcmin region.

The calibration of the NIR polarimetric data is described in Clemens et al. (2012a). The data were reduced using the custom IDL packages Mimir Software Package Basic Data Processing (MSP-BDP) and Photo POLarimetry (MSP-PPOL) (Clemens et al. 2012a). The main reduction and processing steps included taking dome flat fields at each HWP position to correct for variation across the Mimir FOV, accounting for instrumental polarization by observing globular cluster stars, and converting the instrumental polarization position angles to Equatorial coordinates via observations of polarimetric standard stars from Whittet et al. (1992). The resulting combined polarimetric catalog contains the properties of individual stars down to magnitudes of $\sim 13$ for $H$-band and $\sim 14-14.5$ for $K$-band, as measured by Mimir. The polarization position angles, PAs, are measured as the angle East of North in Equatorial coordinates, and can be transformed to Galactic coordinates (Galactic PAs or GPAs) by adding $62.8^{\circ}$.

\subsection{Additional Datasets}

In addition to NIR polarimetry, we used NIR and mid-IR (MIR) photometry from the 2MASS (Skrutskie et al. 2006), UKIDSS (UKIRT Infrared Deep Sky Survey; Lawrence et al. 2007), and GLIMPSE (Benjamin et al. 2003) catalogs, dust continuum data from the Herschel infrared Galactic Plane Survey (Hi-GAL)
(Molinari et al. 2010) and the APEX Telescope Large Area Survey of the Galaxy (ATLASGAL) (Schuller et al. 2009), and molecular line data from the ${ }^{13} \mathrm{CO}$ Galactic Ring Survey (GRS; Jackson et al. 2006). The NIR and MIR photometry were used to estimate the extinctions to the polarization stars. The dust continuum data were used to create an $\mathrm{H}_{2}$ column density map of the cloud. The ${ }^{13} \mathrm{CO}$ data, which have an angular resolution of 46 arcsec, were used to find the gas velocities and line widths in the less dense regions of the cloud.

\section{ANALYSIS AND RESULTS}

Because the B-field of G28.23 can only be probed by stars lying beyond it, we first found the polarimetric stars that were background to the cloud. The relative locations of the stars with respect to the cloud (background, foreground), were determined by comparing the stellar extinctions to the dust emission-traced cloud extinction. We also accounted for foreground extinction and polarizing layers. The column density map of the cloud was found by using the Herschel Hi-GAL and ATLASGAL dust emission data. The background stellar polarization PA orientations were found and compared to the cloud orientation. The cloud volume density was derived from the column density, and used in estimating the plane-of-sky B-field strength. These steps are described in more detail below.

\subsection{Catalog of NIR Polarimetric and Photometric Stars}

A list of NIR stars was created using the 2MASS and UKIDSS photometric data within the $20 \times 20$ arcmin region of Figure 1 . Stars with $H$-band magnitudes brighter than $13^{\text {th }}$ mag were selected from 2MASS, and stars fainter than $13^{\text {th }}$ mag were selected from UKIDSS. Only stars with $H$-band uncertainties less than $0.3 \mathrm{mag}$ were retained. These stars were then matched to the Mimir $H$ and $K$-band polarization catalogs. The number of 2MASS+UKIDSS stars within the $20 \times 20$ arcmin FOV with polarizations in either $H, K$, or both was 17,160. Of these, 3,280 stars had both $H$ and $K$-band polarization entries ( $H$-pol, $K$-pol), while 12,554 stars were found only in $H$-pol and 1,326 were found only in $K$-pol. The number of $H$-pol entries is much higher because the $H$-pol data span a larger area and are located throughout less extincted regions in the FOV than the $K$-band targeted observations. This NIR catalog of stars was then matched to the MIR GLIMPSE catalog. Of the 17,160 NIR stars, 12,490 were positionally-matched to GLIMPSE $4.5 \mu \mathrm{m}$ point sources.

Table 1 lists the polarimetric properties of the stars the polarimetric bands in which the stars were observed, polarization percent, Galactic polarization PAs (GPAs), and Equatorial Stokes $\mathrm{Q}$ and $\mathrm{U}$ parameters $\left(\mathrm{Q}_{E}, \mathrm{U}_{E}\right)$, 
along with uncertainties. The reported GPAs are measured from the North Galactic Pole with GPAs increasing along the East of North direction, where $90^{\circ}$ is parallel to the Galactic plane and 0 and $180^{\circ}$ are perpendicular to the plane. These GPAs were derived by rotating the Equatorial PAs computed from Stokes $\mathrm{U}_{E}$ and $\mathrm{Q}_{E}$ parameters. Table 2 lists the photometric properties of these stars in the same order, including the 2MASS or UKIDSS designation, stellar colors and magnitudes, and the relative extinctions and distances (discussed below).

The polarization percentages reported and used in the analysis have been Ricean corrected (Wardle \& Kronberg 1974) to account for positive bias, where $\sigma_{P}$ is equal to the uncertainty in the polarization percentage:

$$
\begin{array}{r}
P_{\text {uncorrected }}=\sqrt{U_{E}^{2}+Q_{E}^{2}}, \\
P_{\text {corrected }}=\sqrt{P_{\text {uncorrected }}^{2}-\sigma_{P}^{2}} .
\end{array}
$$

All polarization measurements reported in Table 1 and in the results are $\mathrm{P}_{\text {corrected }}$. Stars with $\mathrm{P}_{\text {corrected }}$ signalto-noise ratios (SNR) greater than 2.5 and uncertainties less than $5 \%$ were classified as "high SNR" stars. In the majority of the following analysis, only high SNR stars are used. This selection criteria eliminates faint stars and bright stars with little to no polarization percentages and low uncertainties.

Because many of the stars in the catalog had both $H$ and $K$-pol entries, it was possible to compare the difference in PAs between the two wavelengths $\left(\delta \mathrm{PA}_{K-H}\right)$ for each star. Of the stars with both $H$ and $K$-pol matches, 137 showed high SNR in both bands. Figure 2 shows the distribution of $\delta \mathrm{PA}_{K-H}$ of these high SNR stars. The variance-weighted mean $\delta \mathrm{PA}_{K-H}$ is $-2.1 \pm 0.6^{\circ}$ (this uncertainty estimate includes the observational uncertainty of the weighted mean, $0.23^{\circ}$, added in quadrature to the systematic uncertainty of the Mimir PA measurements of $0.6^{\circ}$, Clemens et al. 2012a), with a weighted standard deviation of $16^{\circ}$. The standard deviation is close to the uncertainty in $\mathrm{PA}$ of a star with polarization signal-to-noise of $2.5\left(11.5^{\circ}\right)$. Therefore, the measured $H$ and $K$-pol PAs are judged to be identical to within their uncertainties.

\subsection{Selecting Background Stars}

To probe the B-field of G28.23, it was necessary to select stars that are background to the cloud. Foreground stars probe material between the cloud and the observer, and therefore, do not probe the cloud B-field. Finding distances to field stars, however, is difficult using only photometric information. Therefore, for the purpose of finding stars background to G28.23, we compared the photometrically determined stellar extinctions to the thermal dust emission-derived cloud column density

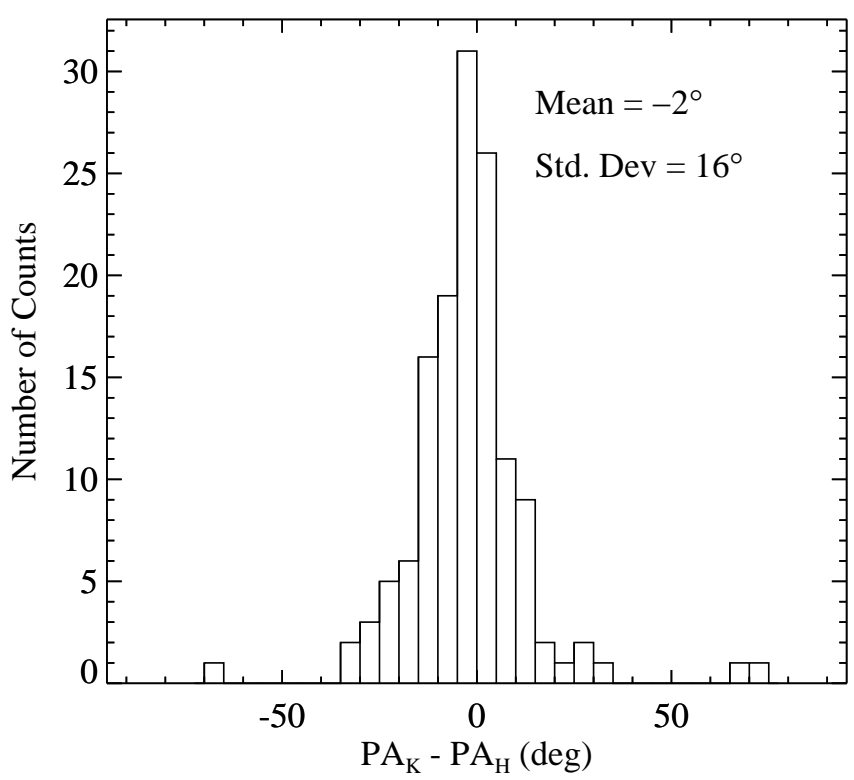

Figure 2. Distribution of the $\delta \mathrm{PA}_{K-H}$ of high SNR stars. The variance-weighted mean and weighted standard deviation of the distribution are $-2^{\circ}$ and $16^{\circ}$, respectively.

(converted to an extinction map) along the same lines of sight. Stars exhibiting larger extinctions than the corresponding cloud extinction were selected as background stars.

\subsubsection{Stellar Extinctions}

The NIR and MIR photometric properties of the polarization stars were used to estimate stellar extinctions. Each polarimetric star in Table 1 contains a photometric entry in Table 2. Not all entries contain a match to the GLIMPSE catalog, as stated in Section 3.1. The intrinsic range of colors for stars at these wavelengths (on the Rayleigh-Jeans tail) is narrow. Therefore, colors observed in excess of the intrinsic values of the stars can be reliably attributed to interstellar extinction. The extinctions of the stars that were matched to GLIMPSE were estimated via the Rayleigh-Jeans Color Excess (RJCE, Majewski et al. 2011) method if their $4.5 \mu \mathrm{m}$ magnitude uncertainties were less than $0.3 \mathrm{mag}$. The RJCE method uses the NIR $H$-band and MIR $4.5 \mu \mathrm{m}$ magnitudes of stars to determine their color excesses, $\mathrm{E}(H-4.5 \mu \mathrm{m})$, following the extinction law of Indebetouw et al. (2005). We adopted an intrinsic $(H-4.5 \mu \mathrm{m})$ 。 equal to $0.08 \mathrm{mag}$ (Majewski et al. 2011) for all stars. For stars that could not be matched to the GLIMPSE catalog, or were matched but their $4.5 \mu \mathrm{m}$ magnitude uncertainties were greater than $0.3 \mathrm{mag}$, we used the Near-Infrared Color Excess (NICE, Lada et al. 1994) method, which uses the $\mathrm{E}(H-K)$ color excesses of stars to estimate their extinctions. We adopted an intrinsic $(H-K)$ 。 of $0.13 \mathrm{mag}$ for these stars. 


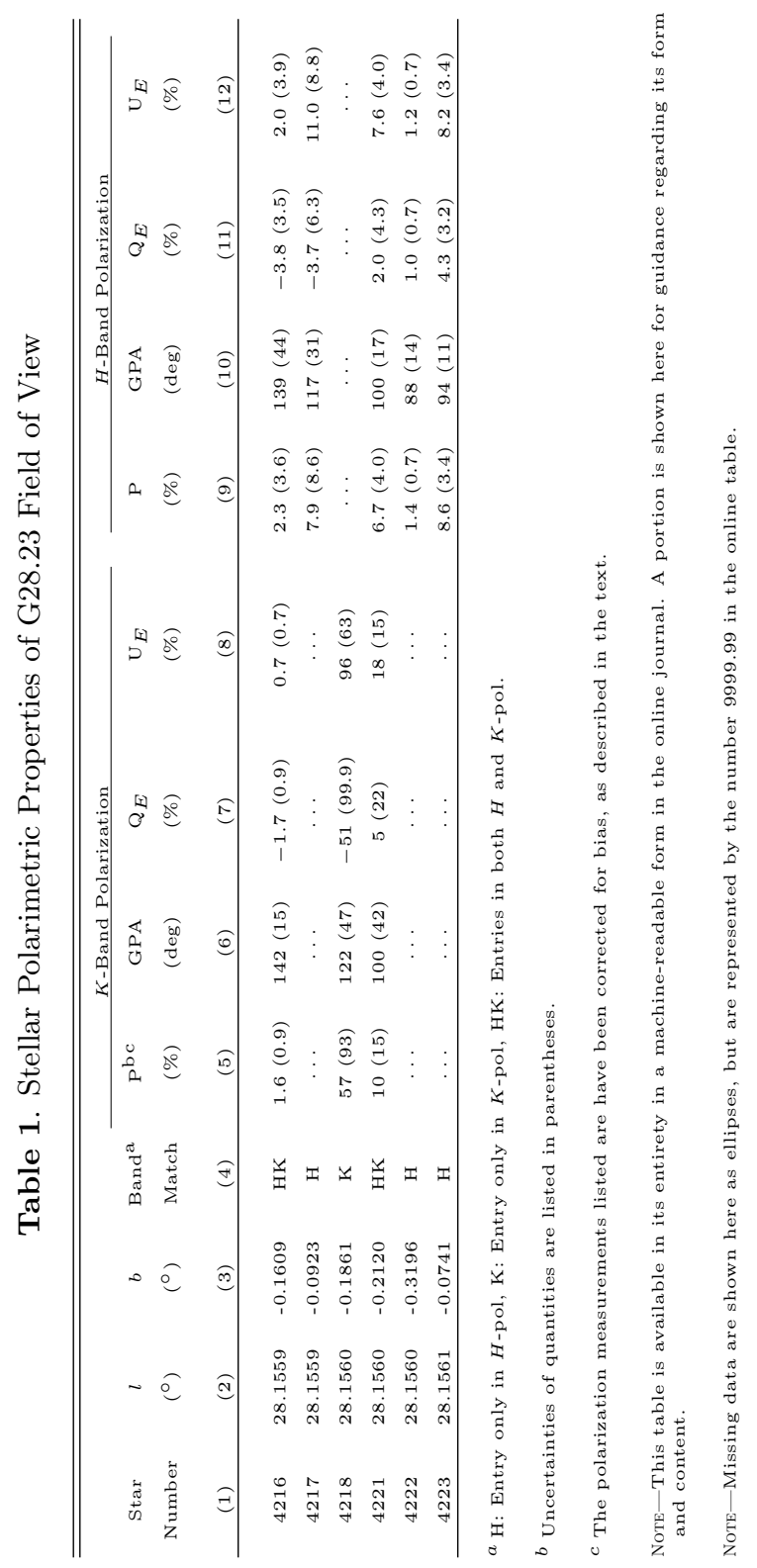




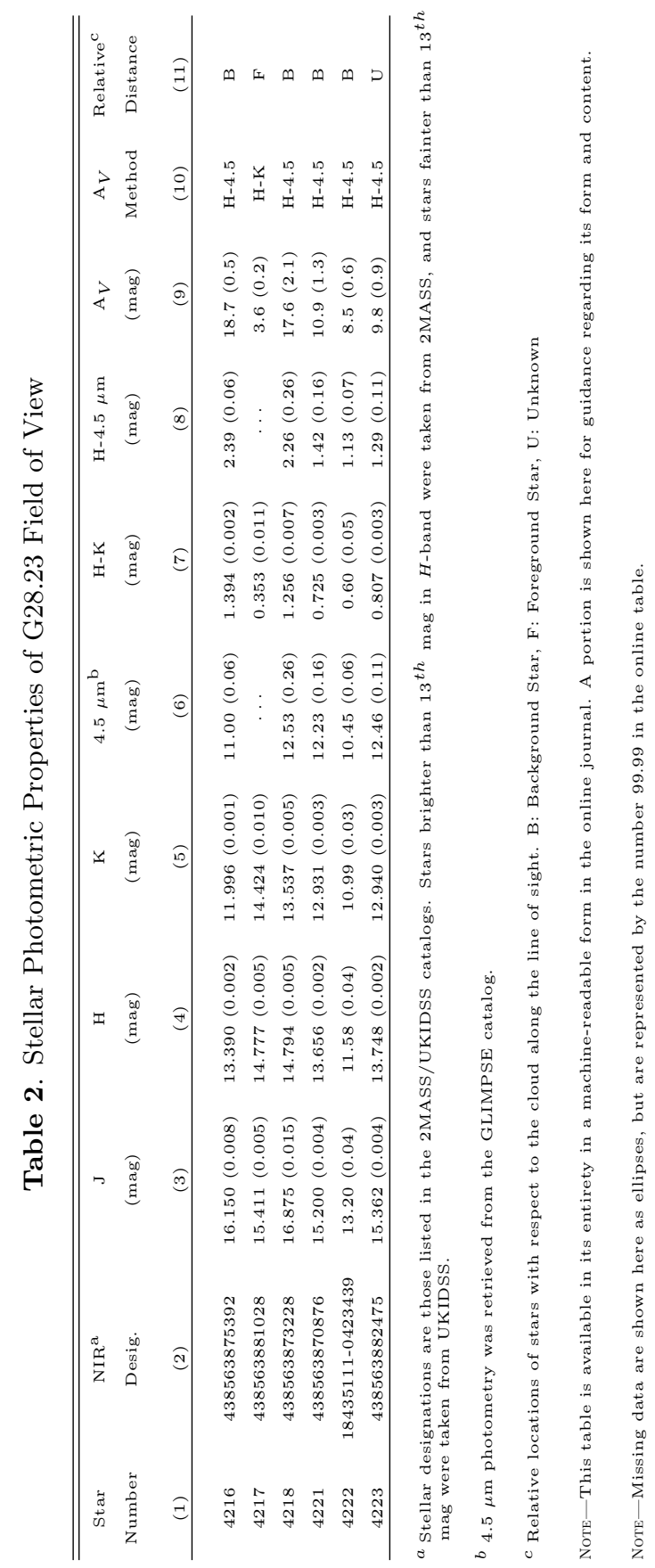




\subsection{Cloud Extinctions}

We used public Herschel data from the Hi-Gal project (Molinari et al. 2010) and data from ATLASGAL (Schuller et al. 2009) to estimate the cloud column density. Herschel data for G28.23 are identified with the obs-ID numbers 1342218694/5 and were observed during April 2011 using the SPIRE+PACS parallel mode, which observed in five wavebands simultaneously (70, $160,250,350$, and $500 \mu \mathrm{m})$. The ATLASGAL data provide additional sub-mm measurements at $870 \mu \mathrm{m}$.

The data were reduced and processed following the procedure described in detail in Guzmán et al. (2015). All maps were convolved to the resolution of the $500 \mu \mathrm{m}$ maps (37 arcsec) and projected to a common pixel grid. To study filamentary IRDCs, it is important to subtract the diffuse far-IR emission of the Galactic plane that is not associated with the filament. A background image, constructed for each Hi-GAL field by smoothing the field image, was subtracted from each field. The subtraction procedure is described in detail in Guzmán et al. (2015) and is similar to that used in other IRDC studies performed using Herschel data (e.g., Battersby et al. 2011). At $500 \mu \mathrm{m}$, the diffuse component subtracted around G28.23 amounts to 250-300 $\mathrm{MJy} \mathrm{sr}^{-1}$, which is comparable to the emission expected from the IRDC itself.

Dust column density and temperature maps were obtained by fitting a single temperature gray-body model to the multiple wavelength intensities of each pixel. These densities and temperatures were converted to gas column densities using theoretical dust opacity curves (Ormel et al. 2011). The dust model used contained no ice coatings and had $3 \times 10^{4}$ years of coagulation for silicate-graphite grains, as might be appropriate for the outer and middle regions of such a dense filament. The uncertainties of the dust temperatures and column densities are given in Guzmán et al. (2015), and are on the order of 10\%. Following Heiderman et al. (2010), the gas column densities were converted to extinctions via the relation $\mathrm{A}_{V}[\mathrm{mag}]=\mathrm{N}_{H_{2}}\left[\mathrm{~cm}^{2}\right] / 1.37 \times 10^{21}\left[\mathrm{~cm}^{2} / \mathrm{mag}\right]$, where $\mathrm{A}_{V}=1.086 \mathrm{C}_{E X T}$ (Draine 2003), $\mathrm{R}_{V}$ is equal to 5.5, and $\mathrm{C}_{E X T}$ at $V$-band is equal to $6.715 \times 10^{-22}$ $\mathrm{cm}^{2} \mathrm{H}^{-1}$ (Weingartner \& Draine 2001) ${ }^{3}$.

Figure 3 shows the GLIMPSE and MIPSGAL 3-color image of G28.23, with column density-based $\mathrm{A}_{V}$ contours overlaid. An $\mathrm{A}_{V}$ of $10 \mathrm{mag}$ corresponds to an $\mathrm{H}_{2}$ column density of $1.37 \times 10^{22} \mathrm{~cm}^{-2}$. The dark extinction feature within the 50 mag $\mathrm{A}_{V}$ contour in Figure 3 corresponds to the densest region of the IRDC. The long axis of the cloud, including both the densest regions of the cloud between longitudes $28^{\circ} .25$ and 28.32 and the less

\footnotetext{
${ }^{3}$ http://www.astro.princeton.edu/ draine/dust/dustmix.html.
}

dense filament at longitudes less than 28.25, extends approximately 12 arcmin. Due to the proximity of G28.23 to the Galactic mid-plane, it is located in a region of non-negligible, and variable, extinction. At latitudes below that of the cloud, farther from the mid-plane, the extinction decreases, whereas at latitudes closer to the midplane, the extinction increases.

\subsubsection{Accounting for Foreground Extinction and Polarization}

Because the dust emission-based cloud extinction estimates accounted for foreground and background extinction unrelated to the IRDC through removal of surrounding diffuse emission, a similar type of correction needed to be done for the stellar extinctions prior to the assignment of stellar locations. To estimate the foreground stellar extinction, we examined the extinctions of the polarization catalog stars that were spatially coincident with the regions of the cloud that had column densities larger than $\mathrm{A}_{V}=30 \mathrm{mag}\left(\mathrm{N}_{H_{2}} \sim 4.11 \times 10^{22} \mathrm{~cm}^{-2}\right)$. These stars are most likely to be foreground stars because, even with deep exposures, it would be very difficult to detect stars in the NIR through such large extinctions.

Figure 4 plots the distribution of stellar extinctions of stars that are listed in the Table 1 polarimetry catalog and are located within the region outlined by the $\mathrm{A}_{V}=30 \mathrm{mag}$ contour in Figure 3. These stars show predominantly low extinctions, with a small tail in the distribution extending to larger extinctions. The binned distribution of extinctions ( $\mathrm{bin}=1 \mathrm{mag}$ ) was fit using a Gaussian plus a constant background. The peak of the fit occurs at 2.06 mag with a Gaussian width $(\sigma)$ of 1.01 mag. Fits made to different bin sizes resulted in similar peaks and widths. Based on the fit, we estimated that the foreground extinction is strongly bounded to be no more than $4.5 \mathrm{mag}$ ( peak mean extinction plus $\sim 2.5 \sigma$ ). This value is a liberal estimate of the foreground extinction, and represents a conservative approach to assigning background stars with high confidence.

All stellar extinctions were estimated following the steps described in Section 3.2.1. Based on their relative extinctions compared to the cloud extinctions at their locations, the stars were classified into three categories: 'foreground,' 'background,' and 'unknown.' Any star with extinction less than $4.5 \mathrm{mag}$ was classified as 'foreground.' The extinctions of the remaining stars were reduced by $4.5 \mathrm{mag}$ and compared to the cloud extinction at the stellar coordinates. Stars with foregroundmodified extinctions larger than the cloud extinction plus cloud extinction uncertainty (10\%) were classified as 'background.' Stars with modified extinctions less than the cloud extinction minus cloud extinction uncertainty were classified as 'foreground.' Stars with extinc- 


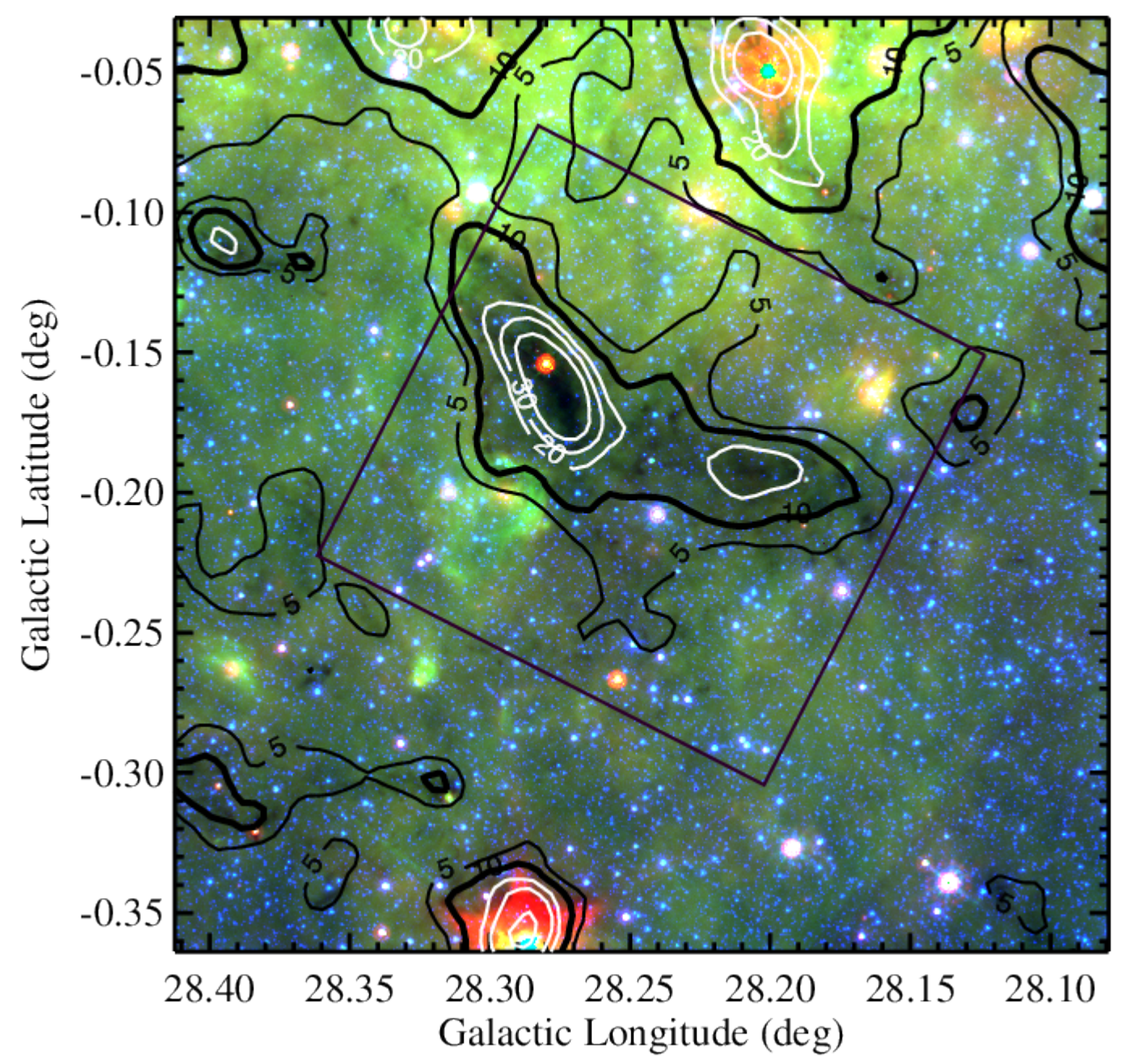

Figure 3. GLIMPSE and MIPSGAL 3-color (blue: 3.6, green: 8, red: $24 \mu \mathrm{m}$ ) background image of G28.23, with column density contours converted to $\mathrm{A}_{V}$ (levels $=[5,10,20,30,50] \mathrm{mag}$ ) overlaid in black and white. The thick black contour of $\mathrm{A}_{V}=10$ mag corresponds to $\mathrm{N}_{H_{2}}=1.37 \times 10^{22} \mathrm{~cm}^{-2}$. The IRDC appears as the dark extinction feature in the center of the contours.

tions that fell within the cloud extinction \pm the cloud extinction uncertainty received distance classifications of 'unknown.' These stars were not included in further analysis because their relative locations with respect to the cloud could not be well-determined.

The estimated stellar extinction (not reduced by 4.5 mag) of each star, along with the extinction estimation method used, and its distance assignment are listed in columns 9, 10, and 11, respectively, in Table 2. The numbers of background, foreground, and unknown stars, with no polarization signal-to-noise cuts applied, were 10597, 6255, and 308, respectively. The numbers of stars that exhibited high polarization SNR in at least one band were 900, 261, and 25 for background, fore- ground, and unknown stars, respectively.

Because foreground material is present along the lineof-sight to G28.23, the polarization measurements of the background stars needed to be corrected for any foreground polarization signal. To remove this effect, we first assumed the foreground polarization was contained in a uniform layer. If more than one significant layer of material was present along the line of sight, the extinction values of the foreground stars would not be prominently peaked around one value. Because this was the case toward the region of G28.23, it was safe to assume one uniform foreground layer.

We computed the variance-weighted average Stokes $\mathrm{U}_{E}$ and $\mathrm{Q}_{E}$ parameters of all foreground stars. This 


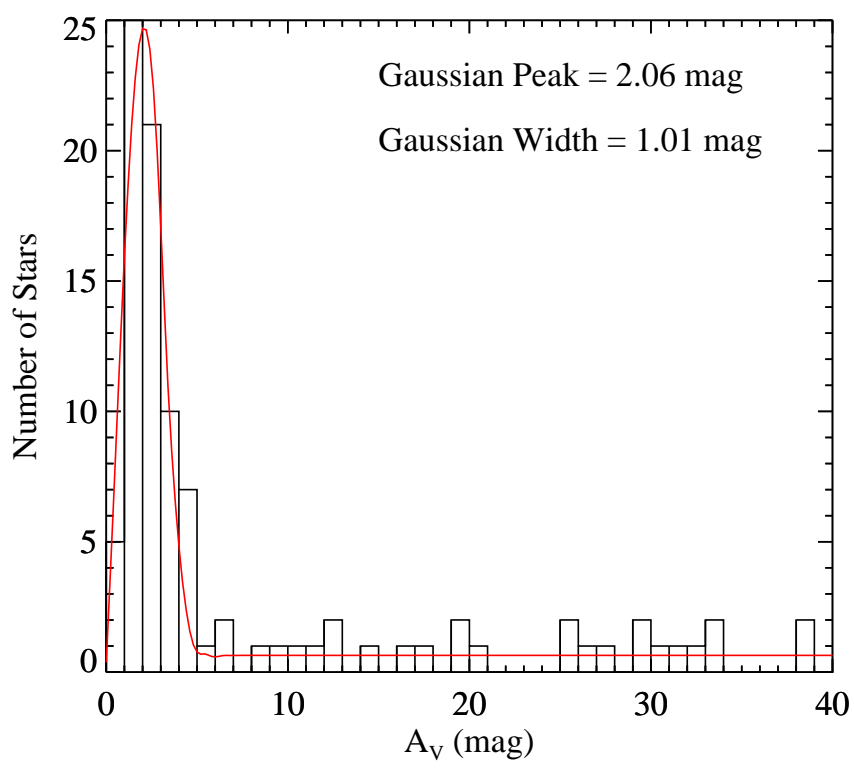

Figure 4. Distribution of estimated visual extinctions of polarization catalog stars projected within the region bounded by the $\mathrm{A}_{V}=30 \mathrm{mag}$ contour of Figure 3. A Gaussian plus a constant fit to the $\mathrm{A}_{V}$ distribution, centered at 2 mag with a width of $1 \mathrm{mag}$, is overlaid in red.

foreground polarization signal was $\sim 0.5 \%$. The average foreground $\mathrm{U}_{E}$ and $\mathrm{Q}_{E}$ values $\left(\bar{U}_{E}=0.40 \%, \bar{Q}_{E}=\right.$ $-0.28 \%$ ) were subtracted from the $\mathrm{U}_{E}$ and $\mathrm{Q}_{E}$ parameters of the individual background stars prior to their use in subsequent analyses. The foreground, weighted U, Q uncertainties (of order $\sim 0.02 \%$ ) were propagated into the foreground-subtracted $\mathrm{U}_{E}$ and $\mathrm{Q}_{E}$ uncertainties of the background stars. We note that the polarization properties of the stars listed in Table 1 have not been foreground-modified, but can be corrected by following the above procedure. The foreground-corrected polarization properties of the background stars are used in the rest of the analyses.

\subsubsection{Properties of the Background Stars}

The number of background stars with a high SNR in $K$-pol was 318 , of which 90 were also detected as high SNR $H$-pol stars. Within the entire $20 \times 20$ arcmin FOV, 574 background stars were detected as high SNR $H$-pol stars, including those 90 stars. Four high SNR $H$-pol stars in the $10 \times 10$ arcmin FOV did not have corresponding $K$-pol entries.

Figure 5 shows a GLIMPSE and MIPSGAL 3-color image of G28.23 overlaid with the dust emission-based column density-derived $\mathrm{A}_{V}$ contours and the high SNR $H$ and $K$ stellar polarization vectors. The polarizations probe to cloud $\mathrm{A}_{V}$ values between 35 to 40 mag. While the $K$-band polarizations cannot probe into the dense, inner regions of the IRDC, where $\mathrm{A}_{V}$ values reach $\sim 100$ mag, they probe to the intermediate densities. Using a combination of $H$-pol and $K$-pol data, we trace the cloud plane-of-sky B-field orientation from the outer diffuse regions into the intermediate extinction regions of the cloud. The large-scale polarization orientation across the FOV is preferentially parallel to the Galactic plane, although some changes to this overall pattern are seen near the IRDC.

The following analysis and results use only the sample of $H$-pol and $K$-pol background stars.

\subsection{Polarization Efficiency}

Because the mechanism of spinning up dust grains to be oriented perpendicular to the intervening B-field relies on an anisotropic radiation field (Lazarian \& Hoang 2007), one concern in using stellar polarimetry to probe B-fields in dense regions is whether the grains remain aligned with the field deep within the cloud (Arce et al. 1998).

A relation between polarization percentage and extinction was found for the polarization stars in the G28.23 $10 \times 10$ arcmin FOV. Figure 6 plots the stellar optical extinction, $\mathrm{A}_{V}$, against the polarization percentage for high SNR $K$-pol stars.

The relation between $\mathrm{A}_{V}$ and polarization was fit by a line, variance-weighted by the polarization percentage uncertainties. The best-fitting line had a slope of $0.059 \pm 0.002 \% \mathrm{mag}^{-1}$. The individual polarization percentages versus stellar extinctions show significant scatter. Therefore, the polarization percentages of the individual stars were binned into extinction bins of width $=2 \mathrm{mag}$, and the variance-weighted average of the stellar polarization percentages in each bin were computed. These binned polarization percentages and the bestfitting line are overlaid in Figure 6 in red. The slope of the best-fitting line to the binned points is $0.060 \pm 0.002$ $\% \mathrm{mag}^{-1}$, which agrees with the slope of the relationship of the individual points.

This positive slope between the $\mathrm{P}_{K}-\mathrm{A}_{V}$ relation is less than the slope derived by Arce et al. (1998) for the polarization measurements of stars in the ISM near the Taurus molecular cloud. Using polarimetric observations taken at $766 \mathrm{~nm}$, they found that $\mathrm{P}_{766 \mathrm{~nm}} \sim 3.58 E_{B-V}$, assuming $\mathrm{A}_{V}=3.1 E_{B-V}$, for $\mathrm{A}_{V}$ less than $1.3 \mathrm{mag}$. Accounting for the expected difference in polarization percentage between the two wavelengths of $766 \mathrm{~nm}$ and $K$-band at $2.2 \mu \mathrm{m}$ following the Serkowski Law, and using an $\mathrm{R}_{V}$ equal to 5.5 , as was done in this study, their relation would be equivalent to $\mathrm{P}_{K} \sim 0.12 \mathrm{~A}_{V}$. This slope is about a factor of two larger than the one derived in this study. The difference between the two slopes may be due to a decrease in polarization efficiency at the large opacities of G28.23, which is embedded in $\sim 2 \mathrm{mag}$ of visual extinction in the Galactic plane. Taurus, on the other hand, is located off the plane, and exposed to the interstellar radiation. 


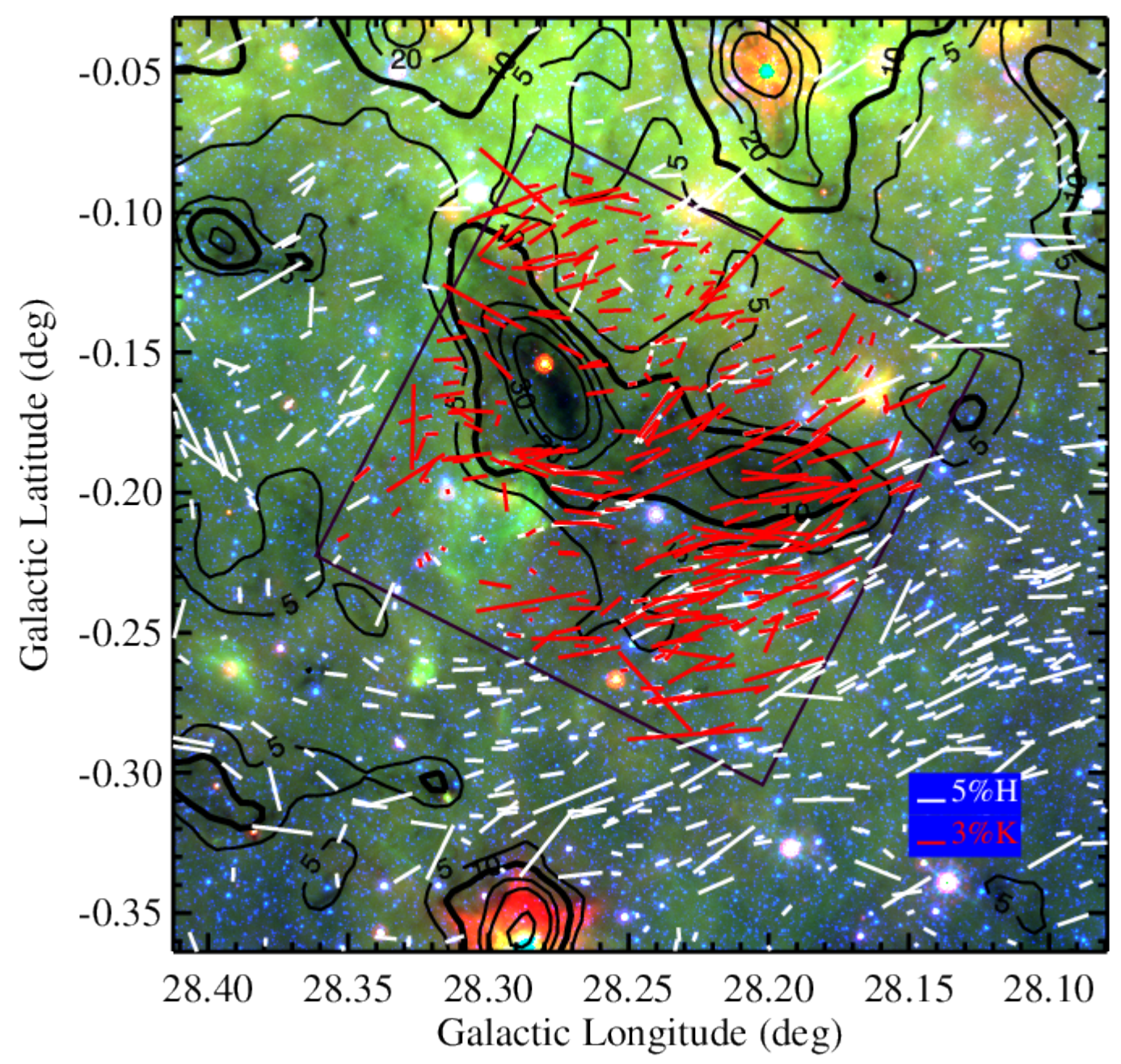

Figure 5. GLIMPSE+MIPSGAL 3-color background image, with column-density-derived $\mathrm{A}_{V}$ contours overlaid in black. The foreground-corrected polarizations of individual high SNR background stars are overlaid in red $(K$-pol) and white $(H$-pol). The lengths of the vectors indicate the polarization percentage, and the scale of the vectors is shown in the bottom right. The $H$-band polarization measurements trace the large-scale behavior of the plane-of-sky B-field, while the $K$-band polarizations probe the B-field of the intermediate densities of the cloud. Ninety stars, which have both $H$ and $K$ high SNR polarization detections, have both white and red vectors shown.

Slopes larger than zero indicate that some polarization signal is being added as higher extinctions are probed. The results obtained here, in answer to Question 1 posed in Section 1.2, imply that the grains remain aligned, at least to some degree, to the highest extinctions probed by the NIR in G28.23.

\subsection{UQ Averaging}

Using only high SNR stars ignores the stars with lower signal-to-noise ratios. While these stars are not significant individually, they can be averaged to boost average polarization signal-to-noise (Clemens et al. 2012b).
Therefore, we spatially averaged the polarimetric information of all of the background stars, separately for the $K$ and $H$-pol stars, to create smoothed $H$ and $K$ polarization maps of the cloud. The maps were gridded into pixels of $30 \times 30$ arcsec, and all stars classified as background were used. The variance-weighted average $\mathrm{U}_{E}$ and $\mathrm{Q}_{E}$ Stokes parameters of the stars located within each pixel were computed (separately for the $H$ and $K$-pol measurements), from which debiased (Section 3.1) average polarization percentages and Equatorial PAs were estimated. The polarizations computed for each pixel were therefore independent. These Equa- 


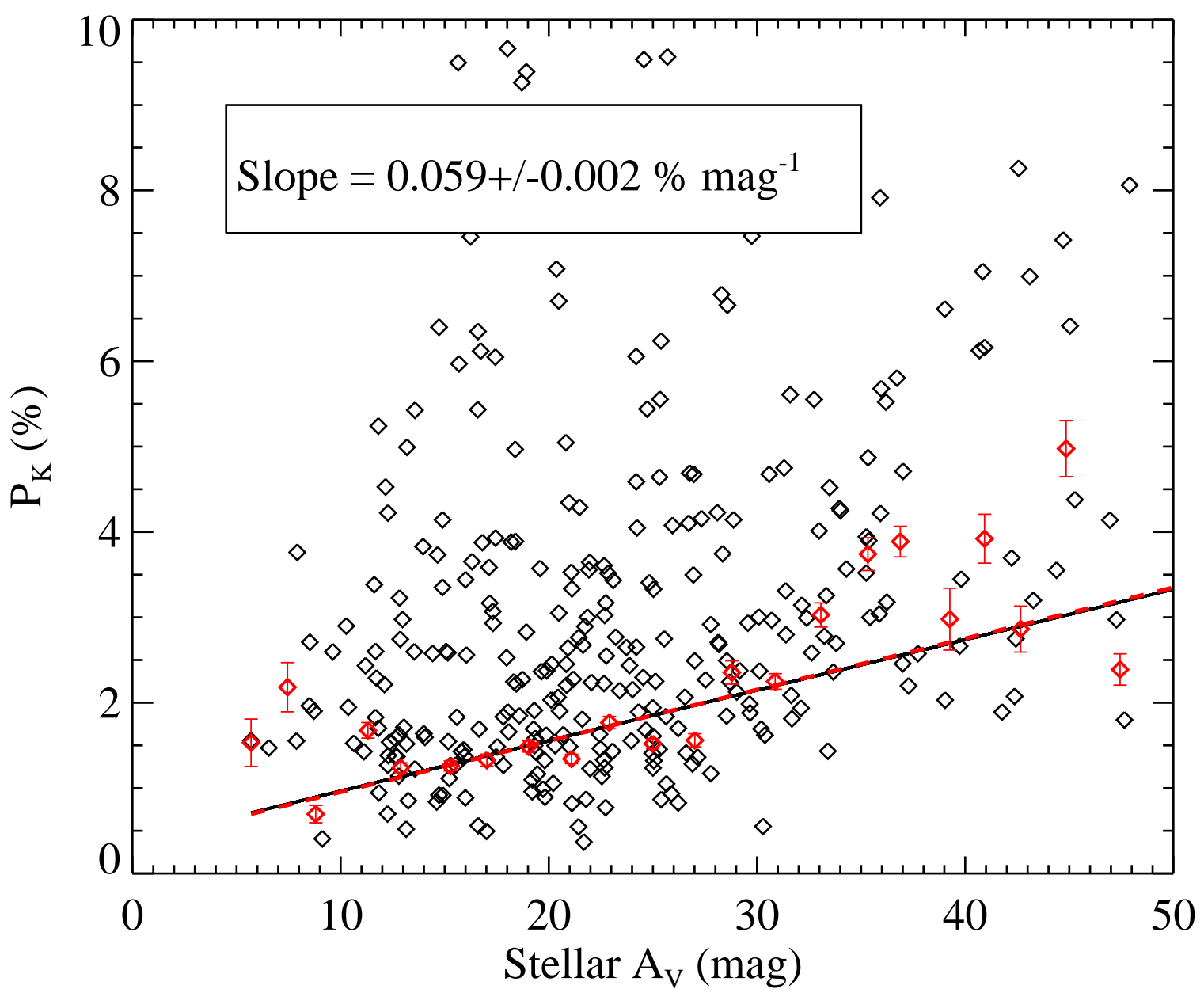

Figure 6. Stellar $K$-band polarization percentage as a function of $\mathrm{A}_{V}$ for the 318 high SNR background $K$-pol stars. The slope of the linear relation between polarization percentage and $\mathrm{A}_{V}$ is greater than zero, which indicates that the polarization percentage increases as a function of increasing extinction. The individual stellar extinctions were separated into 2 mag wide bins, and the weighted average polarization percentage was found in each bin. The binned averages and the best-fitting line between the binned average extinction and polarization percentage are overlaid in red. The slope of this line is equal to 0.06 .

torial PAs were rotated into GPAs.

Figure 7 is similar to Figure 5, but with the spatiallyaveraged polarization vectors plotted. Only the polarization values of pixels where the polarizations were equal to or greater than 2.5 times their propagated uncertainties are shown. In this average polarization map, random components of the polarizations are averaged over, and the large-scale patterns of the polarizations become more evident than in Figure 5. The polarization GPAs are preferentially perpendicular to the cloud major axis farther from the cloud and, especially at larger longitudes, the polarization orientations twist to become more parallel to the cloud major axis as vectors that are closer to the cloud spine are considered.

\subsection{Relative GPA Orientations}

To explore the PA orientation patterns further, we separated the polarization map into four cloud-centered quadrants and compared the GPA distributions of the individual background stars located in each of these regions.

An F-test supported 7th order polynomial was fit to the column density map to define a cloud 'spine' along its long axis (e.g., Marchwinski et al. 2012; Cashman \& Clemens 2014). The polynomial was fit to the pixel locations of the peak values of the column density map along the declination axis, so the spine points were spaced $\sim 37$ arcsec apart. Figure 8 shows the cloud column density-derived $\mathrm{A}_{V}$ contours, with this spine overlaid. The spine splits the cloud into 'Northern' and 'Southern' (Galactic) components. In addition, we also separated the cloud into 'Eastern' and 'Western' sections by using a line running parallel to the R.A. axis through the spine midpoint. This line separates the northern dense region of the cloud, which hosts several massive starless cores (Sanhueza et al. 2013) from the less dense, southern elongated extension of the cloud.

For each high SNR $K$-pol and $H$-pol star in the $10 \times 10$ 


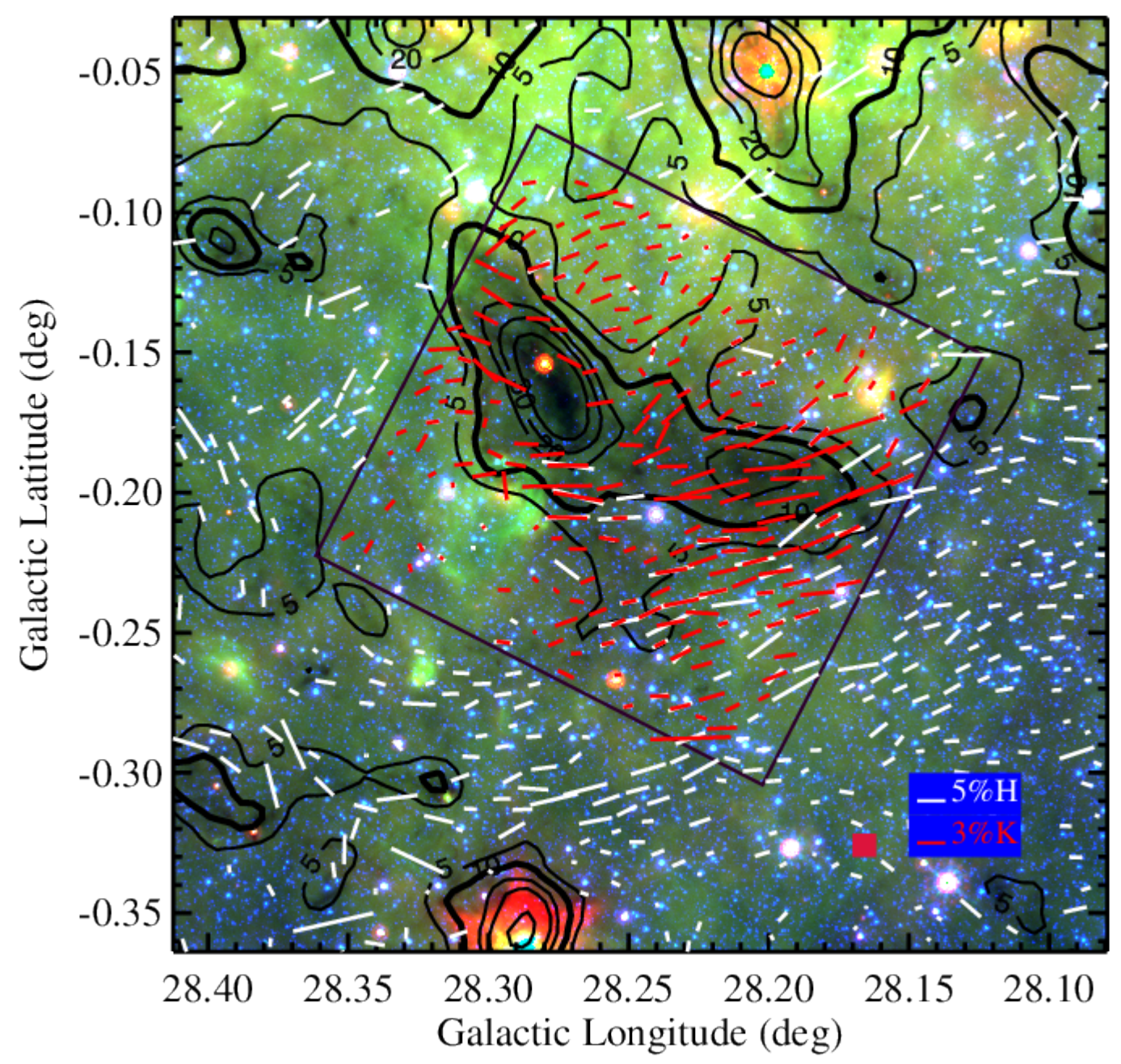

Figure 7. Similar to Figure 5, but with the spatially averaged Stokes $\mathrm{U}_{E}$ and $\mathrm{Q}_{E}$ used to compute the high SNR polarization vectors shown. The $\mathrm{U}_{E}$ and $\mathrm{Q}_{E}$ parameters of the individual stars were averaged over $30 \times 30$ arcsec bins for each of the two wavebands. This bin size is represented by the red box in the bottom right.

arcmin FOV, the PA was found relative to the orientation of the cloud spine at the closest point to the star. The $K$ and $H$-pol PAs were treated as one sample. For stars with both $K$ and $H$-pol measurements, their $K$ band PAs were used, and for stars with a high SNR detection in just one band, the PA of the polarization measurement in that band was used. This method of selecting stellar polarimetric information is hereafter referred to as ' $K$-leading'. The PA differences ranged from 0 to $180^{\circ}$, where $90^{\circ}$ signifies PAs that are perpendicular to the cloud spine, and 0 or $180^{\circ}$ signify PAs that are parallel to the spine. While the difference between two angles with no preferential orientation cannot exceed $90^{\circ}$, a preferential orientation was assigned for the relative PAs between the cloud spine and polarization measurements. This assumption resulted in PA differences between 0 and $180^{\circ}$ instead of 0 and $90^{\circ}$. This assumption was made because angles measured between 0 and $180^{\circ}$ could reveal differences in the relative PA distributions among the four quadrants. Figure 9 shows the distributions of relative PA orientations for stars in the four quadrants.

The four distributions show different relative PA patterns. Standard deviations were calculated for the distributions. Because PA distributions are directional or circular, and wrap around 0 and $180^{\circ}$ (a PA of $190^{\circ}$ is equivalent to $10^{\circ}$ ), the standard deviations of the distributions shown would not necessarily represent the true 


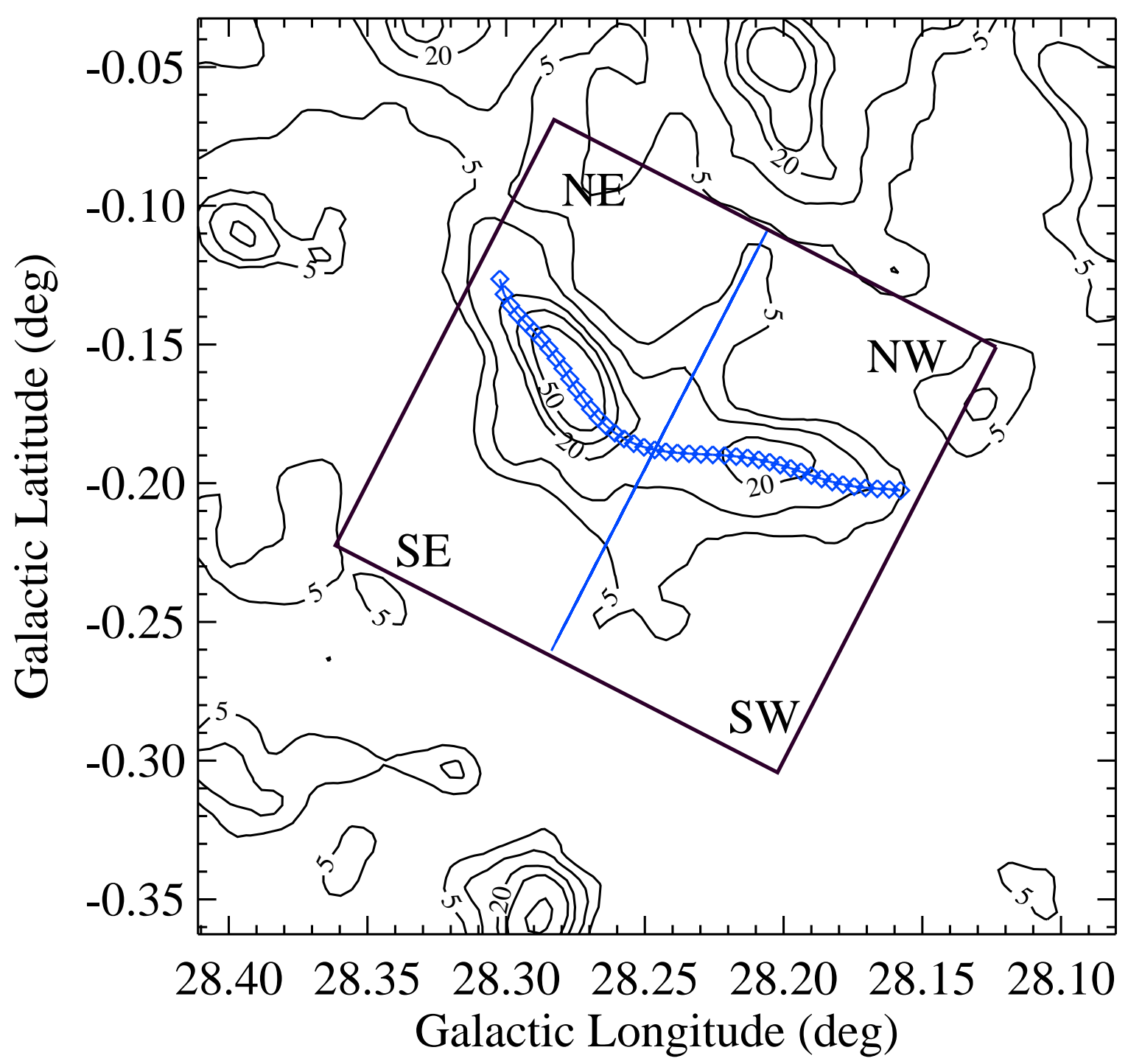

Figure 8. Column-density-derived $\mathrm{A}_{V}$ contours of G28.23 are shown in black, with the box representing the $10 \times 10$ arcmin K-pol FOV overlaid. The spine of the cloud is overlaid as blue diamonds, which separates the cloud into Northern and Southern components. The cloud is further separated into East and West components by a blue line, of constant R.A., running through the spine midpoint.

deviations in the data. The standard deviation of each distribution was found by shifting the whole distribution by increments of $10^{\circ}$ and wrapping PAs greater than $180^{\circ}$, finding the standard deviation of each shifted distribution, and selecting the least deviation. Increments other than $10^{\circ}$ were also tested, but resulted in very similar deviation estimates. The Northeast (larger $l$ and $b$ values) distribution peaks around a relative orientation of $69^{\circ}$ (i.e., more perpendicular) with a standard deviation of $30^{\circ}$. The Southwest distribution peaks around $26^{\circ}$ (more parallel), with a standard deviation of $15^{\circ}$. The Northwest and Southeast distributions are not strongly skewed toward either parallel or perpendicular, though the majority of stars in both distributions have relative PAs between 0 and $90^{\circ}$. The Southeast distri- bution is somewhat more preferentially perpendicular, though it has a larger standard deviation of $36^{\circ}$. Interestingly, there is a large difference in the preferred relative orientations between the Northeast and Northwest quadrants, as well as between the Southeast and Southwest quadrants. The median relative PA of each pair (Northeast to Northwest and Southeast to Southwest) of quadrants differs by nearly $30^{\circ}$.

\subsection{PA Dispersion}

As a partial proxy for the plane-of-sky B-field strength under the $\mathrm{CF}$ method, we calculated the PA dispersion across the cloud. This dispersion calculation used the 322 high SNR $K$ and $H$ polarizations in the $10 \times 10$ arcmin FOV (selecting the polarization information of the stars via the K-leading procedure). 


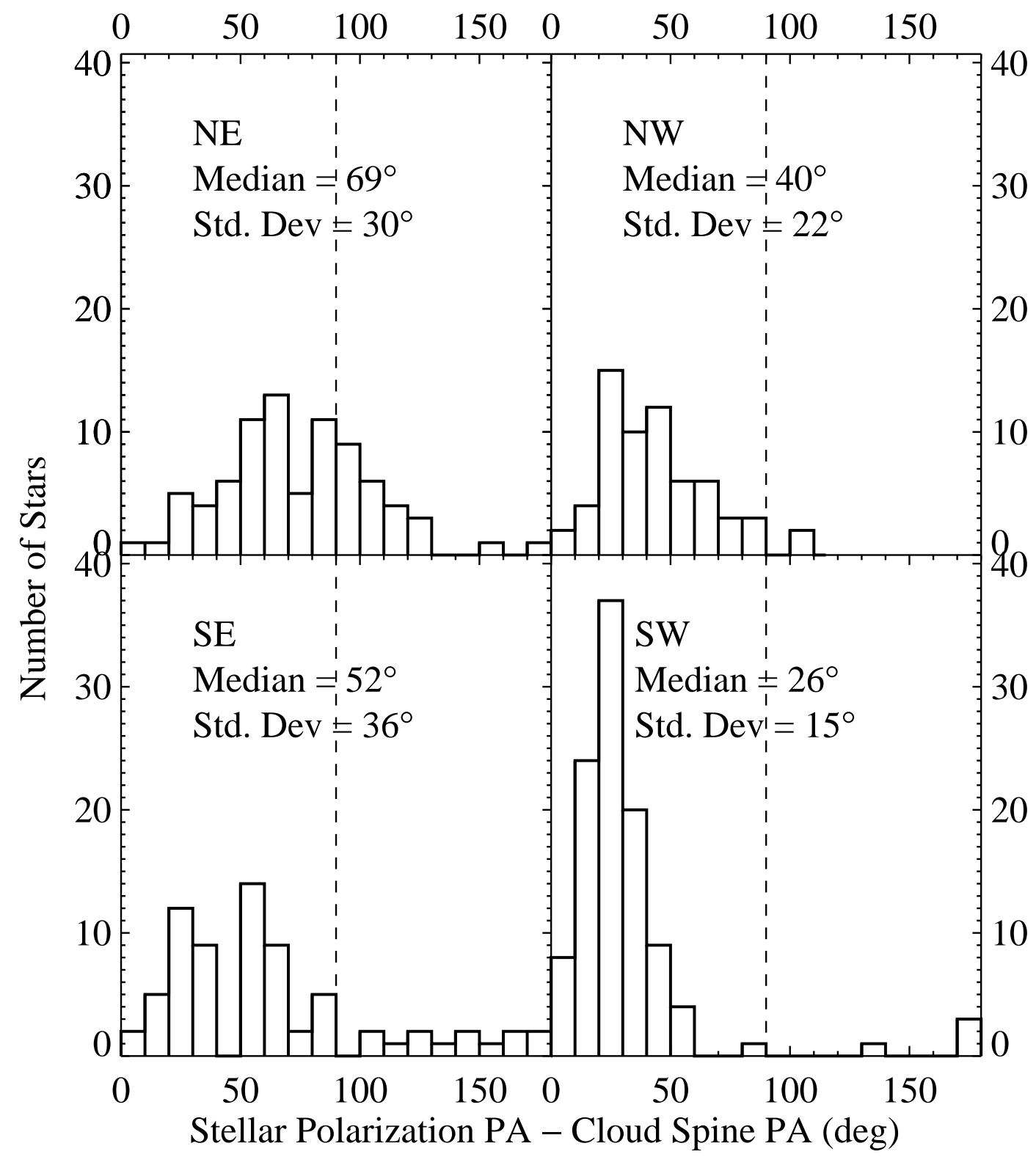

Figure 9. Distributions of polarization PAs of individual high SNR stars relative to the orientation of the spine of G28.23. The four distributions correspond to the quadrants labeled in Figure 8. The median and standard deviation of each distribution are listed. The dashed line in each panel represents $90^{\circ}$, the perpendicular to the cloud orientation.

A large-scale PA pattern was removed from the polarization PAs prior to calculating the PA dispersions, as the estimated dispersion of the B-field should only include the turbulent motions of the gas (e.g., Ostriker et al. 2001). To create the large-scale pattern, the individual stellar PAs ( $K$-leading) were smoothed using variance weighting. Bin centers were separated by 45 $\operatorname{arcsec}$. Smoothing with a Gaussian kernel of $\sigma$ equal to $\sim 38$ arcsec was used to achieve Nyquist sampling. A range of center separations and corresponding Nyquist-sampled Gaussian kernel sizes were tested, but the results did not vary significantly. The resulting smoothed PA map was then interpolated to the size of the Mimir instrument platescale, 0.58 arcsec per pixel. The smoothed and interpolated PA map values were then subtracted from the individual stellar GPAs at the position closest to each star.

The PA dispersions of these large-scale corrected GPAs were calculated using large, overlapping $120 \times 120$ arcsec bins with center separations of 60 arcsec. Such large bins were necessary to ensure that enough stars with high signal-to-noise were used in the dispersion calculations. Dispersions (unweighted standard deviations of the PA distributions) and propagated uncertainties were calculated only for bins with at least seven high SNR stars. The PA distributions were dealiased, as de- 
scribed in Section 3.6, and the standard deviation was found for each iteration. The dispersion was set equal to the minimum standard deviation of the 18 iterations. The uncertainty was the propagated uncertainty of the standard deviation.

Following Hildebrand et al. (2009), the uncertainties of the PA dispersions were subtracted in quadrature from the dispersions. This step was performed to correct the dispersions for the bias added by observational uncertainties, for which the median uncertainty was $\sim 3^{\circ}$. The median post-correction signal-to-noise of the PA dispersions was seven. The PA dispersions and their uncertainties are listed in Table 3. The median PA dispersion was $20^{\circ}$, with a standard deviation of $7^{\circ}$.

The plane-of-sky B-field strengths were calculated using the CF method (described below) in the pixels where the PA dispersions, or the PA dispersions minus their uncertainties, were below $25^{\circ}$, as recommended by Ostriker et al. (2001). The corrected PA dispersion map is shown in Figure 10. The blue regions show where PA dispersions were below $25^{\circ}$. The light gray regions indicate bins where the PA dispersions themselves were above $25^{\circ}$, but the PA dispersions minus their uncertainties were less than or equal to $25^{\circ}$. These blue and light gray regions are where the plane-of-sky B-field strengths were calculated using the CF method . The dispersions could not be calculated in the dense center regions of the cloud due to the lack of stars, which are shown in white. Regions where the PA dispersions minus their uncertainties were above $25^{\circ}$ are shown as dark gray pixels .

\subsection{Cloud Volume Density}

The spatial distribution of cloud volume density is necessary to estimate the plane-of-sky B-field strength using the CF method. The average volume density map of the cloud was derived using the map of dust emissionbased column density. We assumed that the cloud was oriented with its long axis in the plane of the sky.

Before calculating an average volume density map of G28.23, a 3-dimensional (3D) volume density model of the cloud was first created. A normalized column density profile $(\Sigma(r) / \Sigma(0))$, shown in Figure 11, was created using the cloud column density values. The cloud spine was assumed to be located in the plane of the sky (one fixed distance in the line of sight), and the density profile was assumed to be cylindrically symmetric about the cloud spine.

A Plummer-like model was fit to the normalized column density profile, following Equation 1 of Arzouma- nian et al. (2011):

$$
\begin{array}{r}
\rho(\mathbf{r}, k)=\frac{\rho_{c}}{\left[1+\left(\mathbf{r} / R_{\text {flat }}\right)^{2}\right]^{\frac{p}{2}}}, \\
\Sigma(r)=C_{p} \frac{\rho_{\text {ck }} R_{\text {flat }}}{\left[1+\left(r / R_{\text {flat }}\right)^{2}\right]^{\frac{p-1}{2}}}, \\
C_{p}=\int_{-\infty}^{\infty} \frac{\left(1 / R_{\text {flat }}\right) d r}{\left[1+\left(r / R_{\text {flat }}\right)^{2}\right]^{\frac{p}{2}}},
\end{array}
$$

where $k$ is the distance along the cloud spine, $\mathbf{r}$ is the $3 \mathrm{D}$ distance to the cloud spine, $r$ is the projected distance to the closest location, $k$, to the cloud spine, $\rho_{c k}$ is the central gas volume density of the cloud, and $p$ is the profile index. $\mathrm{R}_{\text {flat }}$ represents the radius of the central flat portion of the column density profile. $\Sigma(r)$ is the mass surface density, equal to $\mu \mathrm{m}_{H} N_{H 2}$. The volume density, which depends on the distance from the cloud spine, is $\rho_{p}(\mathbf{r}, k)$. The central density was allowed to vary with $k$. It was constrained by the fit to the column density profile, and was fit point by point along the cloud spine to reproduce the column density values. The best $\mathrm{R}_{\text {flat }}$ and $p$, fit using all of the pixels in the column density map (within the central 10x10 arcmin box in Figure 3), were found to be $0.8 \pm 0.3$ pixels ( $~ 30$ arcsec) and $2.0_{-0.3}^{+0.1}$, respectively. In comparison, the profile index would be equal to 4 for an isothermal cylinder in equilibrium (Ostriker 1964), indicating that there may be some magnetic support for G28.23 (Fiege \& Pudritz 2000). The central densities along the cloud spine were found to range from $4.2 \times 10^{3}$ to $3.1 \times 10^{4} \mathrm{H}_{2} \mathrm{~cm}^{-3}$, with uncertainties of about $200 \mathrm{H}_{2} \mathrm{~cm}^{-3}$.

This method returned a 3D model volume density data cube, with voxel side sizes equal to the column density pixel size $(37$ arcsec $\sim 0.9$ pc at a distance of $5.1 \mathrm{kpc})$.

To calculate an average volume density map, a limiting cloud boundary was needed, since the Plummer profile of the cloud extended to infinity. The cloud boundary was assumed to be located where the column density radial profile reached a plateau. This plateau occurs at an estimated extinction level of $\mathrm{A}_{V} \sim 2 \mathrm{mag}$, indicative of the fact that G28.23 is not an isolated cloud, but is instead embedded in diffuse material. Using the 3D density model, we determined that the $\mathrm{A}_{V}$ contour of $2 \mathrm{mag}$ corresponds to a median volume density of $50 \mathrm{H}_{2} \mathrm{~cm}^{-3}$ in the plane of the sky. Based on this correspondence, the cloud boundary was assumed to be where the maximum volume density along the line of sight just reached $50 \mathrm{H}_{2} \mathrm{~cm}^{-3}$.

To reach an $\mathrm{A}_{V}$ of $2 \mathrm{mag}$ with an average volume density $50 \mathrm{H}_{2} \mathrm{~cm}^{-3}$, a column of $\sim 20 \mathrm{pc}$ is needed, which is comparable to the size of a giant molecular cloud (GMC), and is perhaps indicative of the GMC in which G28.23 is embedded. This $50 \mathrm{H}_{2} \mathrm{~cm}^{-3}$ boundary 


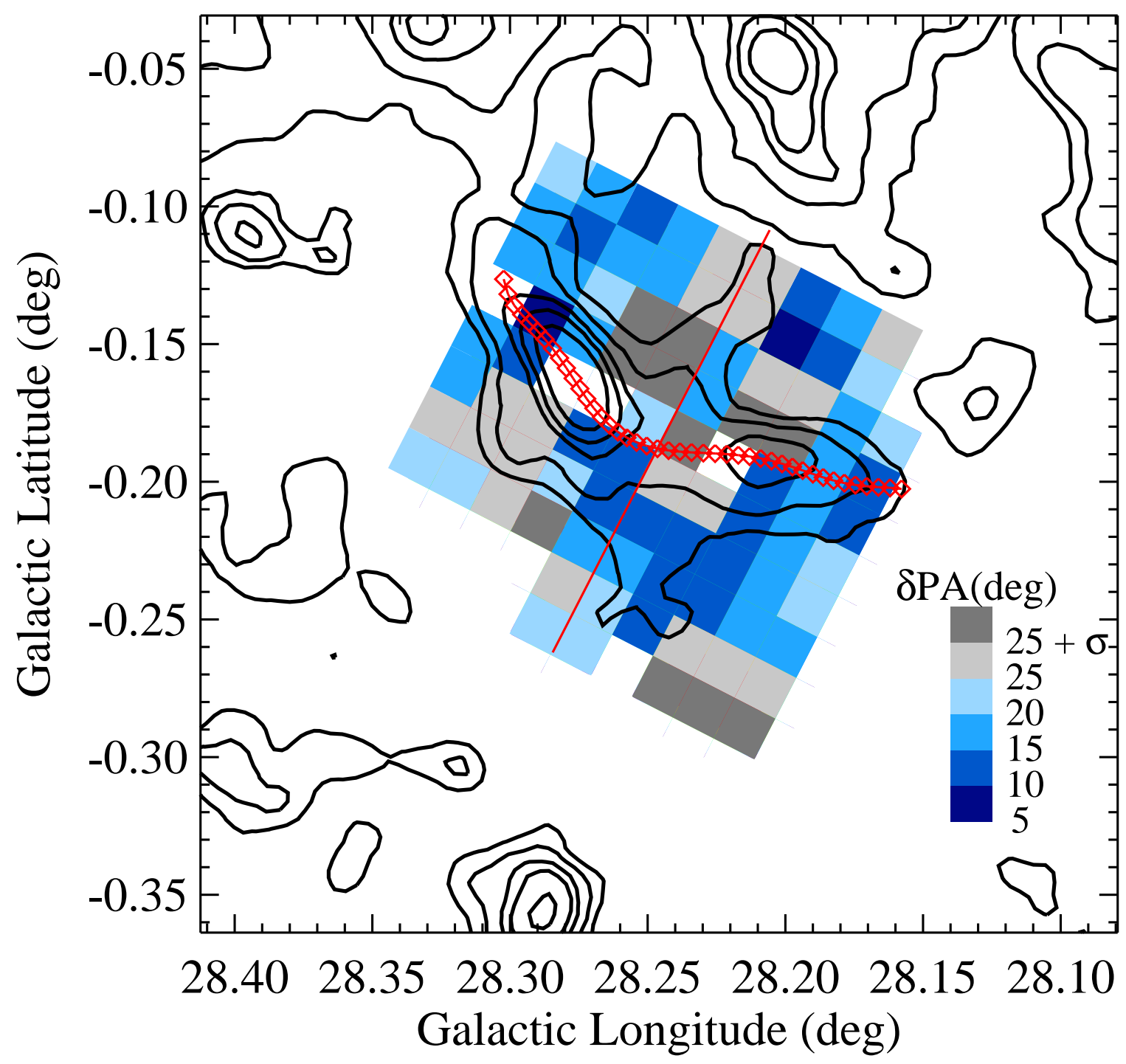

Figure 10. PA dispersion map of G28.23, using only high SNR $H$ and $K$-band stars. For stars with both $H$ and $K$-band high SNR polarization detections, their $K$-band GPAs were used in the dispersion calculations. The blue filled pixels indicate regions where the dispersions are less than $25^{\circ}$, with darker hued-colors corresponding to lower dispersions. Regions of light gray correspond to dispersions that are larger than $25^{\circ}$, but the dispersion values minus their uncertainties are less than or equal to $25^{\circ}$. Dark gray regions indicate where the dispersions minus their uncertainties are larger than $25^{\circ}$, while white regions correspond to locations where the numbers of stars were not sufficient to calculate a dispersion. Column-density derived $\mathrm{A}_{V}$ contours are overlaid in black, and the cloud spine and East-West dividing line are shown in red.

is more liberal than ones used in previous studies, which used the cloud FWHM to estimate the cloud depth along the line of sight (e.g., Crutcher et al. 2004). For G28.23, the FWHM was found by fitting Gaussian profiles to column density slices across the cloud minor axis. The cloud column density FWHM corresponds spatially to a median volume density in the plane of the cloud spine of $\sim 550 \mathrm{H}_{2} \mathrm{~cm}^{-3}$. Using the FWHM boundary in calculating the average volume density map might be overly restrictive in the case of G28.23. The selected boundary of $50 \mathrm{H}_{2} \mathrm{~cm}^{-3}$ corresponds to a cloud width of $\sim 4 \sigma$. The effects of using different boundaries are explored further in Section 4.3.1.
The volume density along each line of sight was averaged for voxels that exhibited density values greater than $50 \mathrm{H}_{2} \mathrm{~cm}^{-3}$. The average $\mathrm{H}_{2}$ volume density map of G28.23 is shown in Figure 12 as concentric filled blue contours. The outermost density boundary indicates where the average volume density just reaches $50 \mathrm{H}_{2} \mathrm{~cm}^{-3}$. The maximum average volume density is $1.9 \times 10^{3} \mathrm{H}_{2} \mathrm{~cm}^{-3}$. If the boundary of $550 \mathrm{H}_{2} \mathrm{~cm}^{-3}$ corresponding to the FWHM of the column density were used instead, the maximum average volume density becomes $6.1 \times 10^{3} \mathrm{H}_{2} \mathrm{~cm}^{-3}$. The larger boundary corresponding to the cutoff of $50 \mathrm{H}_{2} \mathrm{~cm}^{-3}$ allows the B-field across a larger area of the cloud (factor of $\sim 3$ ) to be 


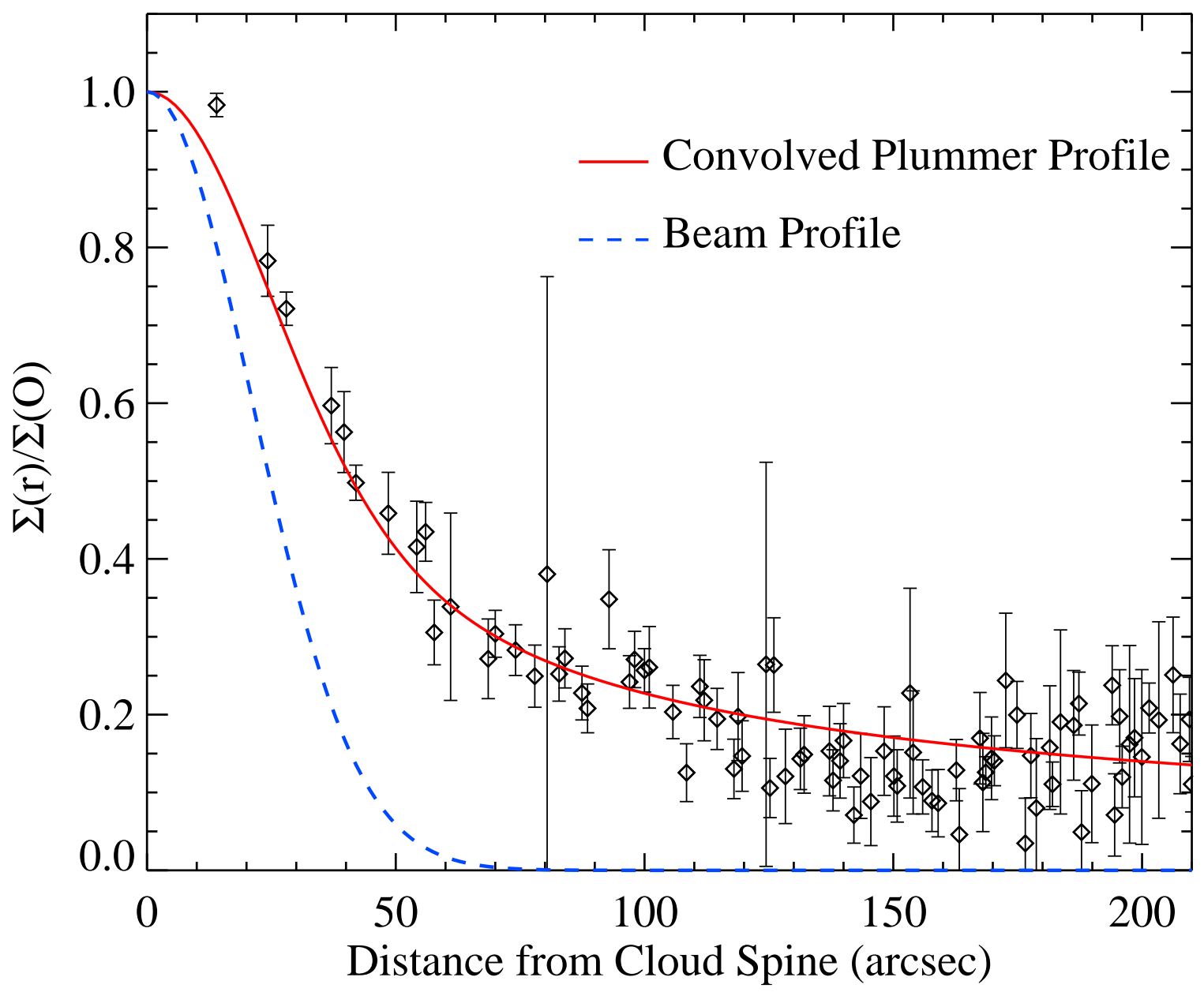

Figure 11. Normalized column density profile of G28.23. Each black diamond represents the median normalized value of the pixels falling into each distance bin in the column density map, where the distance of each pixel is calculated to the closest point on the cloud spine. The error bars enclose \pm 1 standard deviation in each distance bin. A Plummer-like profile was fit to the normalized column density points. The Plummer fit is shown as the solid red line, and the Herschel $500 \mu \mathrm{m}$ beam profile is shown by the dashed blue line. The profile of the column density of the cloud is wider than the Herschel $500 \mu \mathrm{m}$ beam profile.

computed.

\subsection{Plane-of-Sky B-field Strength}

To estimate the plane-of-sky B-field strength $\left(\mathrm{B}_{P O S}\right)$, we used the $\mathrm{CF}$ method, modified as recommended by Ostriker et al. (2001):

$$
B_{P O S}=f \sqrt{4 \pi \rho} \frac{\sigma_{v}}{\sigma_{\phi}},
$$

where $\rho$ is the mass density (in grams $\mathrm{cm}^{-3}$ ), $\sigma_{v}$ is the one-dimensional non-thermal gas velocity dispersion (in $\mathrm{cm} \mathrm{s}^{-1}$ ), $\sigma_{\phi}$ is the polarization PA dispersion (in radians), $f$ is a correction factor, and $B_{P O S}$ is the planeof-sky B-field strength in units of Gauss. Following Ostriker et al. (2001), $f$ is closest to 0.5 , and the method is only valid along directions where the PA dispersion is less than $25^{\circ}$ (0.44 radians).

The gas velocity dispersions were estimated by evaluating the GRS ${ }^{13} \mathrm{CO}$ spectral line data cubes, which have an angular resolution of 46 arcsec (with pixel separations of $23 \mathrm{arcsec}$ ) and spectral resolution of $0.2 \mathrm{~km}$ $\mathrm{s}^{-1}$ (Jackson et al. 2006).

The map of the ${ }^{13} \mathrm{CO}$ integrated intensity was overlaid onto the dust-emission-based column density map to determine whether the ${ }^{13} \mathrm{CO}$ traced the dust column density. The integrated intensity of each pixel in the spectral data cube was calculated by summing the total intensity over $\pm 10 \mathrm{~km} \mathrm{~s}^{-1}$ of the peak radial velocity of $\mathrm{G} 28.23, \sim 80 \mathrm{~km} \mathrm{~s}^{-1}$. Figure 13 shows the ${ }^{13} \mathrm{CO}$ integrated intensity with column density-derived $\mathrm{A}_{V}$ contours overlaid. The ${ }^{13} \mathrm{CO}$ emission at $80 \mathrm{~km} \mathrm{~s}^{-1}$ traces the same region as the dust emission-based column density along the line of sight, indicating that the ${ }^{13} \mathrm{CO}$ spectra can be used to estimate the gas velocity dispersion of the cloud.

The $\mathrm{v}_{l s r}$ of G28.23 peaks at about $81 \mathrm{~km} \mathrm{~s}^{-1}$ (Sanhueza et al. 2013). A secondary smaller peak at 73-34 $\mathrm{km} \mathrm{s}^{-1}$ can be detected in the ${ }^{13} \mathrm{CO}$ spectra in some 


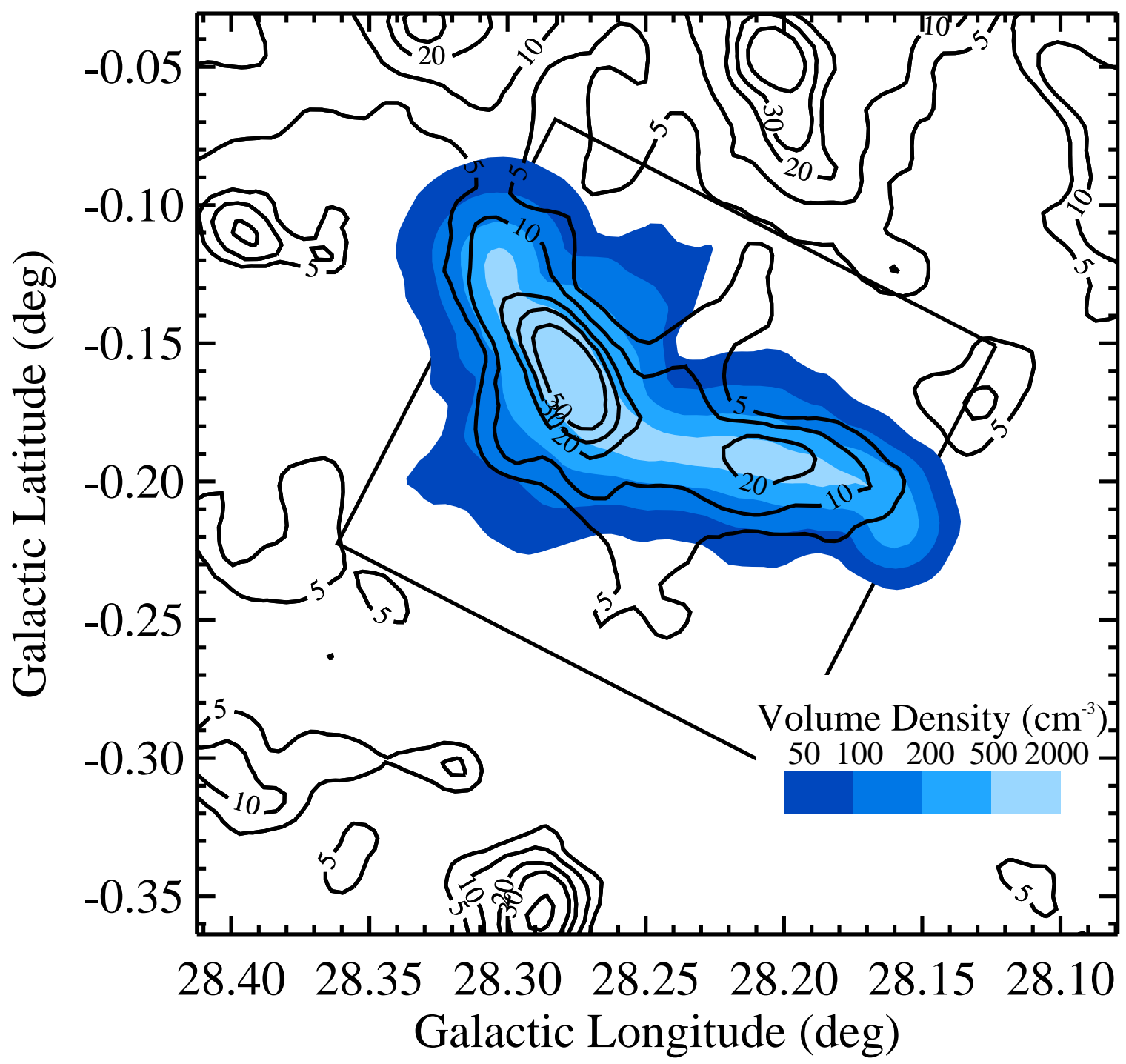

Figure 12. Average volume density map of G28.23, with column-density-derived $A_{V}$ contours overlaid in black. The filled blue contours represent the values of the average volume density, with darker blue hues indicating lower volume density.

regions near the cloud. The $81 \mathrm{~km} \mathrm{~s}^{-1}$ line feature was fit with a Gaussian profile for each GRS spatial pixel falling within the $10 \times 10$ arcmin FOV. Where detected, the secondary component at $73 \mathrm{~km} \mathrm{~s}^{-1}$ was also fit with a Gaussian profile.

Only fit information of the $81 \mathrm{~km} \mathrm{~s}^{-1}$ Gaussian-fitted feature was used in the CF method. The median of the velocity dispersions of the GRS pixels, found from the Gaussian fits, was $\sim 3 \mathrm{~km} \mathrm{~s}^{-1}$, and the median SNR of the velocity dispersions was 12 . The Gaussian sigma fit was interpreted to represent the velocity dispersion, since thermal contributions are negligible for these wide lines.

In regions with high enough densities, ${ }^{13} \mathrm{CO}$ could become optically thick, in which case the line would become saturated and the line width would exceed the gas velocity dispersion. However, the B-field could only be calculated in regions with enough background stellar polarization probes to calculate PA dispersions, which excluded the highest density regions of G28.23. Examination of the ${ }^{13} \mathrm{CO}$ spectra in the regions for which PA dispersions were calculated indicated that the lines were not self-absorbed.

The angular resolutions of the ${ }^{13} \mathrm{CO}$ velocity dispersion, average volume density, and PA dispersion maps were 46 arcsec, 37 arcsec, and 60 arcsec, respectively. Because the PA dispersion angular resolution was the largest of the three, the other two maps needed to be changed to this resolution. The velocity dispersion and volume density maps were regridded to the resolution of the PA dispersion map, with $60 \times 60$ arcsec pixels, where the value of each output pixel was the average of the values of the input pixels that overlapped the output pixel area, weighted by the fraction of the input pixel 


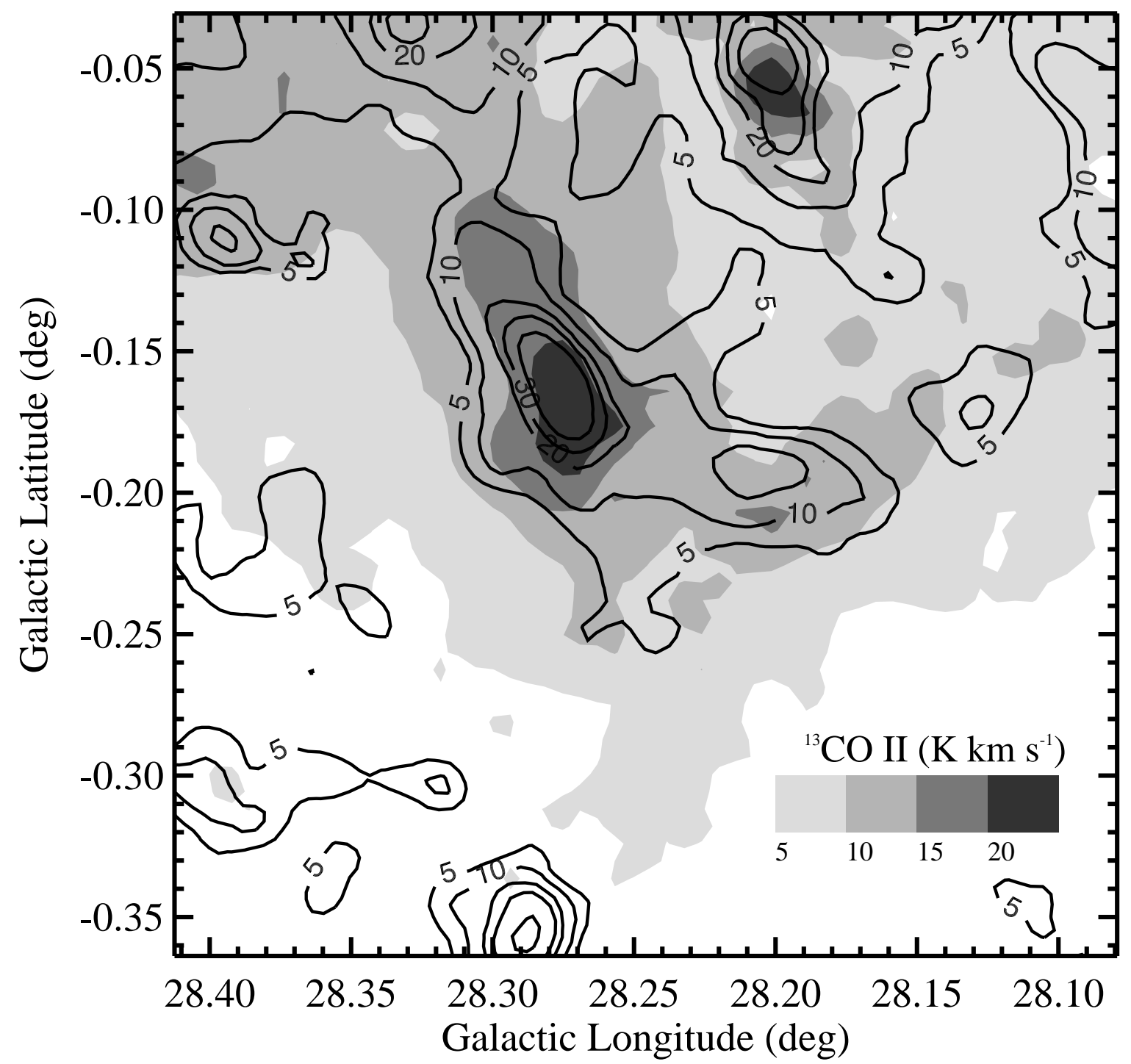

Figure 13. ${ }^{13} \mathrm{CO}$ integrated intensity gray-scale, filled contours with dust-emission based column density-derived $\mathrm{A}_{V}$ contours overlaid. Integrated intensity legend is at bottom right.

area falling into the output pixel.

Equation 6 was used to calculate $\mathrm{B}_{P O S}$ in each of these matched pixels and combined into a map of G28.23. The matched maps consisted of $11 \times 11$ pixels. Of these, $\mathrm{B}_{P O S}$ was calculated in the 51 pixels where PA dispersions, minus their uncertainties, were less than or equal to $25^{\circ}$ and where the volume densities plus their uncertainties were greater than or equal to $50 \mathrm{H}_{2} \mathrm{~cm}^{-3}$. Uncertainties in the $\mathrm{B}_{P O S}$ estimates were found by propagating the uncertainties from the PA dispersions, velocity dispersions, and volume densities. We assumed no uncertainty in the kinematic distance of $5.1 \mathrm{kpc}$. The dependence of the B-field strength on distance (and any distance uncertainty) is discussed in Section 4.3. The $\mathrm{B}_{P O S} \mathrm{SNR}$ values ranged from 1.4 to 6.7 , with a me- dian of 4.2 .

The properties of each bin, including PA dispersion, volume density, ${ }^{13} \mathrm{CO}$ gas velocity dispersion, $\mathrm{B}_{P O S}$, and the normalized Mass-to-Flux ratio (discussed in the following section), along with their uncertainties, are listed in Table 3. The last column of Table 3 lists whether the PA dispersion, density, or both limited the $\mathrm{B}_{P O S}$ calculated for each pixel. If the PA dispersion of a pixel was above $25^{\circ}$, but the dispersion minus its uncertainty was less than or equal to $25^{\circ}$, then the resulting B-field strength calculation yielded an upper limit. Similarly, if the volume density of a pixel was below $50 \mathrm{H}_{2} \mathrm{~cm}^{-3}$, but the density plus its uncertainty was greater than or equal to $50 \mathrm{H}_{2} \mathrm{~cm}^{-3}$, then the resulting B-field strength was also an upper limit. 
The Magnetic Field of IRDC G28.23

Table 3. Properties of the Pixels in the B-field Map

\begin{tabular}{|c|c|c|c|c|c|c|c|}
\hline $\begin{array}{c}l \\
\left({ }^{\circ}\right) \\
(1) \\
\end{array}$ & $\begin{array}{c}b \\
\left({ }^{\circ}\right) \\
(2) \\
\end{array}$ & $\begin{array}{c}\text { PA Dispersion } \\
\text { (deg) } \\
(3) \\
\end{array}$ & $\begin{array}{c}\text { Volume Density } \\
\left.\qquad \mathrm{H}_{2} \mathrm{~cm}^{-3}\right) \\
(4)\end{array}$ & $\begin{array}{c}\sigma_{v}\left({ }^{13} \mathrm{CO}\right) \\
\left(\mathrm{km} \mathrm{s}^{-1}\right) \\
(5)\end{array}$ & $\begin{array}{c}\mathrm{B}_{P O S} \\
(\mu \mathrm{G}) \\
(6) \\
\end{array}$ & $\begin{array}{c}\mathrm{M}_{\|} / \Phi_{\perp} \\
\text { (Normalized) } \\
(7)\end{array}$ & $\begin{array}{c}\text { Limits }^{\mathrm{a}} \\
\text { Used } \\
(8) \\
\end{array}$ \\
\hline 28.1315 & -0.1638 & $\ldots$ & $\ldots$ & $2.1(0.5)$ & $\cdots$ & $\ldots$ & $\ldots$ \\
\hline 28.1463 & -0.1562 & $\ldots$ & $\ldots$ & $2.2(0.3)$ & $\ldots$ & $\ldots$ & $\ldots$ \\
\hline 28.1612 & -0.1486 & $25.9(3.9)$ & $\ldots$ & $2.7(0.2)$ & $\cdots$ & $\ldots$ & $\ldots$ \\
\hline 28.1760 & -0.1410 & $19.7(2.9)$ & $\ldots$ & $3.0(0.2)$ & $\ldots$ & $\ldots$ & $\ldots$ \\
\hline 28.1908 & -0.1334 & $11.8(3.2)$ & $\ldots$ & $2.7(0.5)$ & $\cdots$ & $\cdots$ & $\ldots$ \\
\hline 28.2056 & -0.1258 & $26.1(3.1)$ & $\ldots$ & $2.4(0.2)$ & $\ldots$ & $\ldots$ & $\ldots$ \\
\hline 28.2204 & -0.1181 & $26.1(2.7)$ & $20(9)$ & $2.9(0.4)$ & $\ldots$ & $\ldots$ & $\cdots$ \\
\hline 28.2353 & -0.1105 & $18.9(3.0)$ & $59(25)$ & $2.0(1.8)$ & $17.5(4.9)$ & $1.10(0.33)$ & $\cdots$ \\
\hline 28.2501 & -0.1029 & $13.6(1.9)$ & $57(24)$ & $2.4(0.1)$ & $29.6(7.4)$ & $0.78(0.21)$ & $\ldots$ \\
\hline 28.2649 & -0.0953 & $16.0(2.3)$ & $15(8)$ & $2.3(0.1)$ & $\ldots$ & $\ldots$ & $\ldots$ \\
\hline 28.2797 & -0.0877 & $24.8(5.0)$ & $55(25)$ & $2.4(0.1)$ & $15.6(4.8)$ & $2.88(0.93)$ & $\ldots$ \\
\hline 28.1391 & -0.1787 & $\ldots$ & $12(5)$ & $3.1(0.2)$ & $\ldots$ & $\ldots$ & $\ldots$ \\
\hline 28.1539 & -0.1711 & $\cdots$ & $27(15)$ & $2.5(0.2)$ & $\cdots$ & $\cdots$ & $\cdots$ \\
\hline 28.1688 & -0.1634 & $20.1(2.9)$ & $31(17)$ & $1.7(0.2)$ & $\cdots$ & $\cdots$ & $\cdots$ \\
\hline 28.1836 & -0.1558 & $14.1(2.1)$ & $40(19)$ & $1.4(0.2)$ & $13.5(3.8)$ & $1.51(0.46)$ & $\mathrm{n}$ \\
\hline 28.1984 & -0.1482 & $9.8(2.3)$ & $41(21)$ & $3.8(5.4)$ & $54.8(20.6)$ & $0.49(0.19)$ & $\mathrm{n}$ \\
\hline 28.2132 & -0.1406 & $25.8(2.8)$ & $8(5)$ & $8.4(0.4)$ & $\cdots$ & $\ldots$ & $\ldots$ \\
\hline 28.2281 & -0.1330 & $26.3(2.6)$ & $28(10)$ & $13.3(1.3)$ & $\ldots$ & $\ldots$ & $\ldots$ \\
\hline 28.2429 & -0.1254 & $16.5(3.0)$ & $92(31)$ & $1.9(2.0)$ & $24.9(6.8)$ & $0.62(0.18)$ & $\ldots$ \\
\hline 28.2577 & -0.1177 & $16.2(2.3)$ & $100(33)$ & $1.6(0.1)$ & $22.0(4.8)$ & $0.57(0.14)$ & $\ldots$ \\
\hline 28.2725 & -0.1101 & $14.6(2.1)$ & $90(35)$ & $2.7(0.1)$ & $39.5(9.5)$ & $0.78(0.20)$ & $\cdots$ \\
\hline 28.2874 & -0.1025 & $19.6(3.4)$ & $111(37)$ & $2.5(0.1)$ & $30.1(7.2)$ & $2.51(0.65)$ & $\cdots$ \\
\hline 28.1467 & -0.1935 & $\cdots$ & $117(42)$ & $3.6(0.3)$ & $\cdots$ & $\cdots$ & $\cdots$ \\
\hline 28.1616 & -0.1859 & $20.7(3.3)$ & $132(44)$ & $3.4(0.2)$ & $42.0(9.7)$ & $1.26(0.32)$ & $\cdots$ \\
\hline 28.1764 & -0.1783 & $18.9(2.7)$ & $122(40)$ & $3.3(0.3)$ & $42.6(9.3)$ & $1.36(0.33)$ & $\cdots$ \\
\hline 28.1912 & -0.1706 & $27.3(2.9)$ & $117(39)$ & $5.9(0.5)$ & $51.5(10.3)$ & $0.50(0.11)$ & $\mathrm{PA}$ \\
\hline 28.2060 & -0.1630 & $26.5(2.8)$ & $100(34)$ & $5.3(0.7)$ & $43.7(8.8)$ & $0.28(0.06)$ & PA \\
\hline 28.2209 & -0.1554 & $16.6(2.3)$ & $68(28)$ & $3.4(0.5)$ & $36.9(9.1)$ & $1.56(0.41)$ & $\ldots$ \\
\hline 28.2357 & -0.1478 & $30.6(1.8)$ & $39(17)$ & $2.9(4.2)$ & $\cdots$ & $\cdots$ & $\cdots$ \\
\hline 28.2505 & -0.1402 & $29.0(2.1)$ & $142(43)$ & $1.3(0.1)$ & $\cdots$ & $\cdots$ & $\cdots$ \\
\hline 28.2653 & -0.1326 & $20.0(2.8)$ & $190(54)$ & $2.2(0.1)$ & $33.7(6.8)$ & $1.25(0.28)$ & $\cdots$ \\
\hline 28.2802 & -0.1249 & $18.1(2.2)$ & $217(63)$ & $2.3(0.1)$ & $40.9(7.8)$ & $1.84(0.39)$ & $\cdots$ \\
\hline 28.2950 & -0.1173 & $18.3(2.0)$ & $249(71)$ & $2.1(0.1)$ & $39.4(10.7)$ & $3.69(1.06)$ & $\cdots$ \\
\hline 28.1544 & -0.2083 & $\cdots$ & $404(87)$ & $2.9(0.6)$ & $\cdots$ & $\cdots$ & $\cdots$ \\
\hline 28.1692 & -0.2007 & $14.7(2.6)$ & $461(96)$ & $3.2(0.2)$ & $102.1(21.1)$ & $1.18(0.27)$ & $\cdots$ \\
\hline 28.1840 & -0.1931 & $15.0(2.0)$ & $521(108)$ & $3.5(0.3)$ & $116.6(20.0)$ & $1.71(0.34)$ & $\ldots$ \\
\hline 28.1988 & -0.1855 & $29.0(3.1)$ & 469 (106) & $6.4(0.5)$ & $\cdots$ & $\cdots$ & $\cdots$ \\
\hline 28.2137 & -0.1779 & $31.5(3.6)$ & $283(82)$ & $5.7(0.6)$ & $\cdots$ & $\cdots$ & $\cdots$ \\
\hline 28.2285 & -0.1702 & $17.7(2.2)$ & $147(45)$ & $4.5(0.5)$ & $68.1(13.6)$ & $1.76(0.39)$ & $\cdots$ \\
\hline 28.2433 & -0.1626 & $32.2(1.9)$ & $121(38)$ & $4.1(0.5)$ & $\cdots$ & $\cdots$ & $\cdots$ \\
\hline 28.2581 & -0.1550 & $37.2(2.4)$ & $261(72)$ & $1.9(0.1)$ & $\cdots$ & $\cdots$ & $\cdots$ \\
\hline 28.2730 & -0.1474 & $16.4(4.1)$ & $516(134)$ & $2.5(0.1)$ & $76.0(15.6)$ & $4.91(1.12)$ & $\cdots$ \\
\hline 28.2878 & -0.1398 & $8.6(2.6)$ & $888(182)$ & $2.1(0.1)$ & $163.1(24.4)$ & $3.17(0.57)$ & $\cdots$ \\
\hline 28.3026 & -0.1322 & $\cdots$ & $672(135)$ & $1.9(0.1)$ & $\cdots$ & $\cdots$ & $\cdots$ \\
\hline 28.1620 & -0.2231 & $\cdots$ & $191(57)$ & $2.3(1.7)$ & $\cdots$ & $\cdots$ & $\cdots$ \\
\hline
\end{tabular}


Table 3 (continued)

\begin{tabular}{|c|c|c|c|c|c|c|c|}
\hline $\begin{array}{c}l \\
\left({ }^{\circ}\right) \\
(1) \\
\end{array}$ & $\begin{array}{l}b \\
\left({ }^{\circ}\right) \\
(2) \\
\end{array}$ & $\begin{array}{c}\text { PA Dispersion } \\
\text { (deg) } \\
(3) \\
\end{array}$ & $\begin{array}{c}\text { Volume Density } \\
\qquad \begin{array}{c}\left(\mathrm{H}_{2} \mathrm{~cm}^{-3}\right) \\
(4)\end{array} \\
\end{array}$ & $\begin{array}{c}\sigma_{v}\left({ }^{13} \mathrm{CO}\right) \\
\left(\mathrm{km} \mathrm{s}^{-1}\right) \\
(5) \\
\end{array}$ & $\begin{array}{c}\mathrm{B}_{P O S} \\
(\mu \mathrm{G}) \\
(6) \\
\end{array}$ & $\begin{array}{c}\mathrm{M}_{\|} / \Phi_{\perp} \\
\text { (Normalized) } \\
(7) \\
\end{array}$ & $\begin{array}{c}\text { Limits }^{\mathrm{a}} \\
\text { Used } \\
(8) \\
\end{array}$ \\
\hline 28.1768 & -0.2155 & $14.4(3.0)$ & $155(50)$ & $2.3(0.2)$ & 44.1 (11.8) & $1.79(0.51)$ & $\ldots$ \\
\hline 28.1916 & -0.2079 & $15.8(2.0)$ & $287(79)$ & $3.3(0.2)$ & 77.7 (14.6) & $1.63(0.35)$ & $\cdots$ \\
\hline 28.2064 & -0.2003 & $14.7(2.7)$ & 473 (108) & $3.1(0.2)$ & $99.9(21.5)$ & $2.36(0.56)$ & $\ldots$ \\
\hline 28.2213 & -0.1927 & $\cdots$ & $610(125)$ & $2.4(0.5)$ & . & . & $\ldots$ \\
\hline 28.2361 & -0.1851 & $29.5(3.0)$ & 535 (110) & $2.6(0.8)$ & $\ldots$ & $\ldots$ & $\ldots$ \\
\hline 28.2509 & -0.1774 & $24.9(2.7)$ & $508(113)$ & $3.5(1.2)$ & $68.8(11.0)$ & $2.97(0.56)$ & $\ldots$ \\
\hline 28.2657 & -0.1698 & $\ldots$ & $835(174)$ & $3.0(0.0)$ & .. & .. & $\cdots$ \\
\hline 28.2806 & -0.1622 & $\ldots$ & $1192(238)$ & $2.6(0.1)$ & $\ldots$ & $\ldots$ & $\ldots$ \\
\hline 28.2954 & -0.1546 & $12.2(3.0)$ & 649 (158) & $2.7(0.1)$ & 123.8 & $3.54(0.82)$ & $\ldots$ \\
\hline 28.3102 & -0.1470 & $16.5(2.9)$ & $271(78)$ & $2.3(0.1)$ & $49.8(11.4)$ & $2.48(0.62)$ & ... \\
\hline 28.1696 & -0.2380 & $\cdots$ & $43(19)$ & $2.0(0.2)$ & $\ldots$ & $\ldots$ & $\mathrm{n}$ \\
\hline 28.1844 & -0.2304 & $21.2(3.3)$ & $46(23)$ & $4.2(0.3)$ & $29.6(8.6)$ & $0.90(0.28)$ & $\mathrm{n}$ \\
\hline 28.1992 & -0.2227 & $19.0(2.5)$ & $96(34)$ & $3.2(0.2)$ & $36.0(8.0)$ & $1.35(0.33)$ & $\cdots$ \\
\hline 28.2141 & -0.2151 & $12.9(1.7)$ & $127(40)$ & $3.1(0.2)$ & $60.3(12.4)$ & $1.55(0.35)$ & $\ldots$ \\
\hline 28.2289 & -0.2075 & $27.1(3.3)$ & $161(50)$ & $1.6(0.2)$ & $16.6(3.3)$ & $6.40(1.42)$ & PA \\
\hline 28.2437 & -0.1999 & $26.3(3.3)$ & $271(75)$ & $1.2(1.9)$ & $16.6(4.0)$ & $7.51(1.97)$ & PA \\
\hline 28.2585 & -0.1923 & $11.8(2.0)$ & $485(112)$ & $2.9(8.5)$ & $120.2(42.6)$ & $1.39(0.51)$ & $\ldots$ \\
\hline 28.2734 & -0.1847 & $12.5(2.8)$ & $601(141)$ & $2.9(0.1)$ & $125.8(31.9)$ & $2.81(0.77)$ & $\ldots$ \\
\hline 28.2882 & -0.1770 & $26.3(2.2)$ & $399(107)$ & $3.0(0.1)$ & $49.8(7.9)$ & $6.32(1.18)$ & PA \\
\hline 28.3030 & -0.1694 & $26.4(2.3)$ & $242(67)$ & $3.7(0.1)$ & $48.5(7.9)$ & $2.68(0.51)$ & PA \\
\hline 28.3178 & -0.1618 & $15.4(3.5)$ & $113(36)$ & $3.0(0.2)$ & 45.0 & $0.95(0.28)$ & $\ldots$ \\
\hline 28.1772 & -0.2528 & $\cdots$ & $\ldots$ & $3.0(0.3)$ & $\ldots$ & $\ldots$ & $\ldots$ \\
\hline 28.1920 & -0.2452 & $23.6(3.4)$ & $4(2)$ & $4.6(0.5)$ & $\ldots$ & $\ldots$ & $\cdots$ \\
\hline 28.2069 & -0.2376 & $19.8(2.2)$ & $36(19)$ & $4.5(0.5)$ & $30.2(9.0)$ & $0.09(0.03)$ & $\mathrm{n}$ \\
\hline 28.2217 & -0.2299 & $12.3(1.3)$ & $52(23)$ & $5.7(0.8)$ & $73.3(18.0)$ & $0.45(0.12)$ & $\ldots$ \\
\hline 28.2365 & -0.2223 & $10.1(1.5)$ & $61(27)$ & $3.0(0.3)$ & 50.7 (13.7) & $1.23(0.36)$ & $\cdots$ \\
\hline 28.2513 & -0.2147 & $12.5(2.5)$ & $90(33)$ & $2.2(0.2)$ & $36.3(9.9)$ & $2.17(0.63)$ & $\cdots$ \\
\hline 28.2662 & -0.2071 & $13.7(2.2)$ & $122(41)$ & $2.9(1.0)$ & $51.2(12.0)$ & $2.09(0.53)$ & $\ldots$ \\
\hline 28.2810 & -0.1995 & $23.4(2.8)$ & $171(53)$ & $3.5(0.2)$ & $43.5(8.5)$ & $2.73(0.60)$ & $\ldots$ \\
\hline 28.2958 & -0.1919 & $33.6(9.5)$ & $155(46)$ & $3.1(0.1)$ & $25.2(8.0)$ & $4.11(1.37)$ & PA \\
\hline 28.3106 & -0.1842 & $28.1(8.9)$ & $135(40)$ & $3.5(0.2)$ & 32.1 (11.2) & $3.06(1.11)$ & PA \\
\hline 28.3255 & -0.1766 & $25.6(3.2)$ & $80(30)$ & $6.2(0.5)$ & $47.7(10.8)$ & $0.50(0.12)$ & PA \\
\hline 28.1848 & -0.2676 & $\cdots$ & $\ldots$ & $6.2(0.7)$ & $\ldots$ & $\ldots$ & $\ldots$ \\
\hline 28.1996 & -0.2600 & $16.7(4.2)$ & $\ldots$ & $8.3(0.8)$ & $\ldots$ & $\cdots$ & $\cdots$ \\
\hline 28.2145 & -0.2524 & $17.5(2.3)$ & ... & $5.3(0.5)$ & $\cdots$ & .. & $\ldots$ \\
\hline 28.2293 & -0.2448 & $13.5(1.8)$ & $\cdots$ & $5.4(0.5)$ & $\cdots$ & $\cdots$ & $\cdots$ \\
\hline 28.2441 & -0.2372 & $10.6(2.4)$ & $1(0)$ & $4.2(0.5)$ & $\cdots$ & $\cdots$ & $\cdots$ \\
\hline 28.2589 & -0.2295 & $15.3(2.7)$ & $22(11)$ & $3.4(0.6)$ & .. & .. & $\ldots$ \\
\hline 28.2738 & -0.2219 & $18.2(2.7)$ & $54(24)$ & $3.0(0.2)$ & $26.6(7.1)$ & $1.45(0.41)$ & $\ldots$ \\
\hline 28.2886 & -0.2143 & $34.5(4.4)$ & $90(31)$ & $3.3(0.1)$ & $\ldots$ & $\ldots$ & $\ldots$ \\
\hline 28.3034 & -0.2067 & $34.9(17.9)$ & $87(30)$ & $2.7(0.1)$ & $15.6(8.5)$ & $1.19(0.65)$ & PA \\
\hline 28.3182 & -0.1991 & $22.1(15.5)$ & $84(29)$ & $5.2(0.3)$ & $47.8(34.5)$ & $0.61(0.45)$ & $\cdots$ \\
\hline 28.3331 & -0.1915 & $24.4(4.9)$ & $67(25)$ & $11.7(0.7)$ & $86.8(23.9)$ & $0.31(0.09)$ & $\cdots$ \\
\hline 28.1924 & -0.2824 & $\cdots$ & $\cdots$ & $9.0(0.6)$ & $\cdots$ & $\cdots$ & $\cdots$ \\
\hline 28.2073 & -0.2748 & $27.0(3.8)$ & $\cdots$ & $11.2(1.1)$ & $\cdots$ & $\cdots$ & $\cdots$ \\
\hline 28.2221 & -0.2672 & $25.7(2.6)$ & $\cdots$ & $6.6(0.4)$ & $\cdots$ & . & $\ldots$ \\
\hline 28.2369 & -0.2596 & $25.3(2.6)$ & $\ldots$ & $6.1(0.5)$ & $\ldots$ & $\ldots$ & $\ldots$ \\
\hline
\end{tabular}


The Magnetic Field of IRDC G28.23

Table 3 (continued)

\begin{tabular}{|c|c|c|c|c|c|c|c|}
\hline $\begin{array}{l}l \\
\left({ }^{\circ}\right) \\
(1) \\
\end{array}$ & $\begin{array}{l}b \\
\left({ }^{\circ}\right) \\
(2) \\
\end{array}$ & $\begin{array}{c}\text { PA Dispersion } \\
\text { (deg) } \\
(3) \\
\end{array}$ & $\begin{array}{c}\text { Volume Density } \\
\qquad \begin{array}{c}\left(\mathrm{H}_{2} \mathrm{~cm}^{-3}\right) \\
(4)\end{array}\end{array}$ & $\begin{array}{c}\sigma_{v}\left({ }^{13} \mathrm{CO}\right) \\
\left(\mathrm{km} \mathrm{s}^{-1}\right) \\
(5) \\
\end{array}$ & $\begin{array}{c}\mathrm{B}_{P O S} \\
(\mu \mathrm{G}) \\
(6) \\
\end{array}$ & $\begin{array}{c}\mathrm{M}_{\|} / \Phi_{\perp} \\
\text { (Normalized) } \\
(7) \\
\end{array}$ & $\begin{array}{c}\text { Limits }^{\mathrm{a}} \\
\text { Used } \\
(8) \\
\end{array}$ \\
\hline 28.2517 & -0.2520 & $11.1(2.6)$ & $\ldots$ & $3.2(0.2)$ & $\ldots$ & $\ldots$ & $\ldots$ \\
\hline 28.2666 & -0.2444 & $21.5(2.6)$ & $\ldots$ & $2.8(0.2)$ & $\ldots$ & $\ldots$ & $\ldots$ \\
\hline 28.2814 & -0.2367 & $26.2(3.0)$ & $4(2)$ & $2.2(0.1)$ & $\ldots$ & $\cdots$ & $\cdots$ \\
\hline 28.2962 & -0.2291 & $\ldots$ & $49(24)$ & $2.2(0.1)$ & $\ldots$ & $\ldots$ & $\mathrm{n}$ \\
\hline 28.3110 & -0.2215 & $\ldots$ & $55(29)$ & $1.8(0.1)$ & $\ldots$ & $\ldots$ & $\ldots$ \\
\hline 28.3259 & -0.2139 & $\cdots$ & $41(20)$ & $8.0(1.5)$ & $\ldots$ & & $\mathrm{n}$ \\
\hline 28.3407 & -0.2063 & $\cdots$ & $47(23)$ & $7.9(12.4)$ & $\cdots$ & $\cdots$ & $\mathrm{n}$ \\
\hline 28.2000 & -0.2973 & $\ldots$ & $\ldots$ & $3.9(0.3)$ & $\ldots$ & $\ldots$ & $\cdots$ \\
\hline 28.2149 & -0.2896 & $33.2(3.2)$ & $\ldots$ & $5.3(0.7)$ & $\ldots$ & $\ldots$ & $\cdots$ \\
\hline 28.2297 & -0.2820 & $32.5(2.7)$ & $0(0)$ & $4.9(0.4)$ & $\ldots$ & $\ldots$ & $\ldots$ \\
\hline 28.2445 & -0.2744 & $31.8(4.2)$ & $0(0)$ & $10.4(0.8)$ & $\ldots$ & $\ldots$ & $\ldots$ \\
\hline 28.2593 & -0.2668 & $\ldots$ & $0(0)$ & $6.8(0.6)$ & $\cdots$ & $\cdots$ & $\cdots$ \\
\hline 28.2742 & -0.2592 & $22.8(3.8)$ & $\ldots$ & $2.3(0.1)$ & $\ldots$ & $\ldots$ & $\ldots$ \\
\hline 28.2890 & -0.2516 & $20.6(3.6)$ & $\cdots$ & $2.0(0.1)$ & $\ldots$ & $\cdots$ & $\cdots$ \\
\hline 28.3038 & -0.2440 & $\ldots$ & $\cdots$ & $1.6(0.1)$ & $\cdots$ & $\cdots$ & $\cdots$ \\
\hline 28.3187 & -0.2363 & $\ldots$ & $\ldots$ & $1.7(0.1)$ & $\ldots$ & $\ldots$ & $\cdots$ \\
\hline 28.3335 & -0.2287 & $\cdots$ & & $7.9(18.3)$ & & & $\cdots$ \\
\hline 28.3483 & -0.2211 & $\cdots$ & $\cdots$ & $6.5(14.3)$ & $\cdots$ & $\cdots$ & $\cdots$ \\
\hline 28.2077 & -0.3121 & $\cdots$ & $\ldots$ & $8.7(0.8)$ & $\cdots$ & $\ldots$ & $\cdots$ \\
\hline 28.2225 & -0.3045 & $\cdots$ & $\ldots$ & $5.3(0.5)$ & $\ldots$ & $\ldots$ & $\cdots$ \\
\hline 28.2373 & -0.2968 & $\ldots$ & $\ldots$ & $6.9(0.8)$ & $\ldots$ & $\ldots$ & $\ldots$ \\
\hline 28.2521 & -0.2892 & $\ldots$ & $\ldots$ & $14.9(1.8)$ & $\ldots$ & $\ldots$ & $\ldots$ \\
\hline 28.2670 & -0.2816 & $\cdots$ & $\cdots$ & $11.4(1.1)$ & $\cdots$ & $\cdots$ & $\cdots$ \\
\hline 28.2818 & -0.2740 & $\ldots$ & $\ldots$ & $4.3(0.3)$ & $\ldots$ & $\ldots$ & $\ldots$ \\
\hline 28.2966 & -0.2664 & $\cdots$ & $\cdots$ & $2.6(0.2)$ & $\cdots$ & $\ldots$ & $\cdots$ \\
\hline 28.3114 & -0.2588 & $\ldots$ & $\ldots$ & $2.4(0.2)$ & $\ldots$ & $\ldots$ & $\cdots$ \\
\hline 28.3263 & -0.2512 & $\ldots$ & $\ldots$ & $2.9(0.2)$ & $\ldots$ & $\ldots$ & $\cdots$ \\
\hline 28.3411 & -0.2435 & $\ldots$ & & $4.2(0.3)$ & & & $\ldots$ \\
\hline 28.3559 & -0.2359 & $\ldots$ & 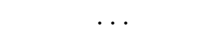 & $1.8(2.1)$ & $\cdots$ & $\cdots$ & $\cdots$ \\
\hline
\end{tabular}

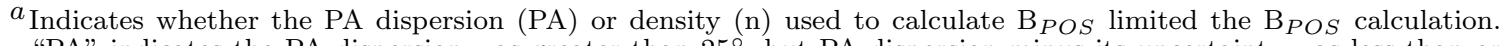
"PA" indicates the PA dispersion was greater than $25^{\circ}$, but PA dispersion minus its uncertainty was less than or equal to $25^{\circ}$. " $\mathrm{n}$ " indicates that the volume density was less than $50 \mathrm{H}_{2} \mathrm{~cm}^{-3}$, but the density plus its uncertainty was greater than or equal to $50 \mathrm{H}_{2} \mathrm{~cm}^{-3}$.

Figure 14 presents the B-field strengths in map form, where the derived field strengths range from $10-165 \mu \mathrm{G}$, and probe extinctions up to $\mathrm{A}_{V} \sim 30$ mag. The field strength estimates are predominantly lower in the lower density regions of the upper left (NE) quadrant of Figure 14. The field strength could not be probed in the densest regions of the cloud because of the lack of NIR background stars with polarimetric detections.

\subsubsection{B-field Strength versus Density}

One test of whether a B-field could have influenced the flow of material into a cloud is determining the dependence of the field strength on cloud density. This relation between B-field strength and average volume density is shown for G28.23 in Figure 15, which is based on, and uses data from, Figure 1 of Crutcher et al. (2010). The $\mathrm{B}_{P O S}$ strengths of G28.23 were scaled to represent the mean B-field strength in only one dimension $\left(B_{x}=B_{P O S} / \sqrt{2}\right)$, and are shown as the black diamonds. The volume density $(n)$ used in this relation is the volume density of atomic hydrogen, found by doubling the molecular hydrogen volume density. The Zeeman measurements and upper limits from Crutcher et al. (2010) are shown as the blue triangles. Also shown is a red, dashed line representing the equation of $\mathrm{B}_{\max }$ from 


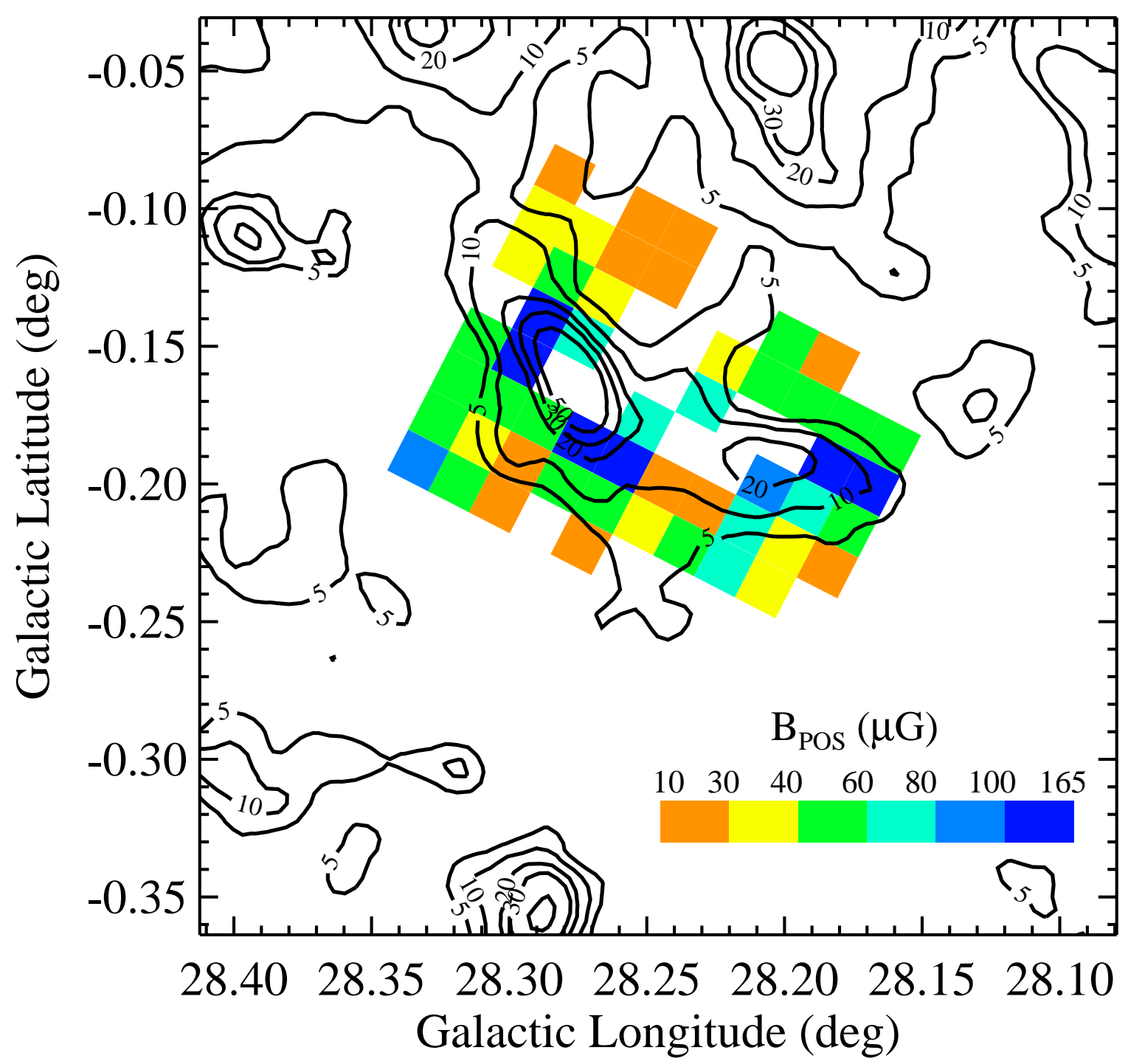

Figure 14. Plane-of-sky B-field strength for G28.23, estimated using the CF Method. Black solid lines show the column-densityderived $\mathrm{A}_{V}$ contours. Filled pixels show the $\mathrm{B}_{P O S}$ values, as indicated by the color bar. Missing pixels failed to meet the PA dispersion and/or volume density criteria.

their Equation 21. Most of the $\mathrm{B}_{x}$ values for G28.23 exceed the corresponding $\mathrm{B}_{\max }$ values at the given densities. We note that the $\mathrm{B}_{x}$ points of $\mathrm{G} 28.23$ are for a single cloud, while the $\mathrm{B}_{Z}$ points of Crutcher et al. (2010) consist of one point per cloud for various samples of clouds and Zeeman probes.

A single power law of the form $B \sim n^{\alpha}$ was fit to the G28.23 $\mathrm{B}_{x}$ versus $n$ points. The best-fit power law had a slope $\alpha=0.73 \pm 0.06$ (black solid line in Figure 15). The implications of this result will be discussed in Section 4.2 .

\subsection{Mass-to-Flux Ratio}

The $\mathrm{M} / \Phi_{B}$ of a region determines whether gravitational or magnetic energy dominates (Crutcher 2012). The normalized $\mathrm{M} / \Phi_{B}\left(\mathrm{M} / \Phi_{B N}\right)$, is equal to (Crutcher et al. 2004):

$$
M / \Phi_{B N}=7.6 \times 10^{-21} N_{H_{2}} / B
$$

where $\mathrm{N}_{H_{2}}$ is in $\mathrm{cm}^{-2}$ and $\mathrm{B}$ is in $\mu \mathrm{G}$. A normalized $\mathrm{M} / \Phi_{B}$ equal to unity indicates that gravitational energy is equal to the magnetic energy.

We calculated the normalized $\mathrm{M} / \Phi_{B}$ of the regions of G28.23 where $\mathrm{B}_{P O S}$ values were calculated (filled pixels in Figure 14). The B-field strength used in the $\mathrm{M} / \Phi_{B N}$ calculation is nominally $\mathrm{B}_{T O T}$, which is the amplitude of the 3-D vector B-field strength (Crutcher et al. 2004). However, because we measure $\mathrm{B}_{P O S}$, the $\mathrm{M} / \Phi_{B N}$ calculated here is actually $\mathrm{M}_{\|} / \Phi_{\perp}$ (Planck Collaboration et al. 2016b), with the input B-field strength being $\mathrm{B}_{P O S}$. Based on the geometry of the cloud and whether the B-field is perpendicular or parallel to the 


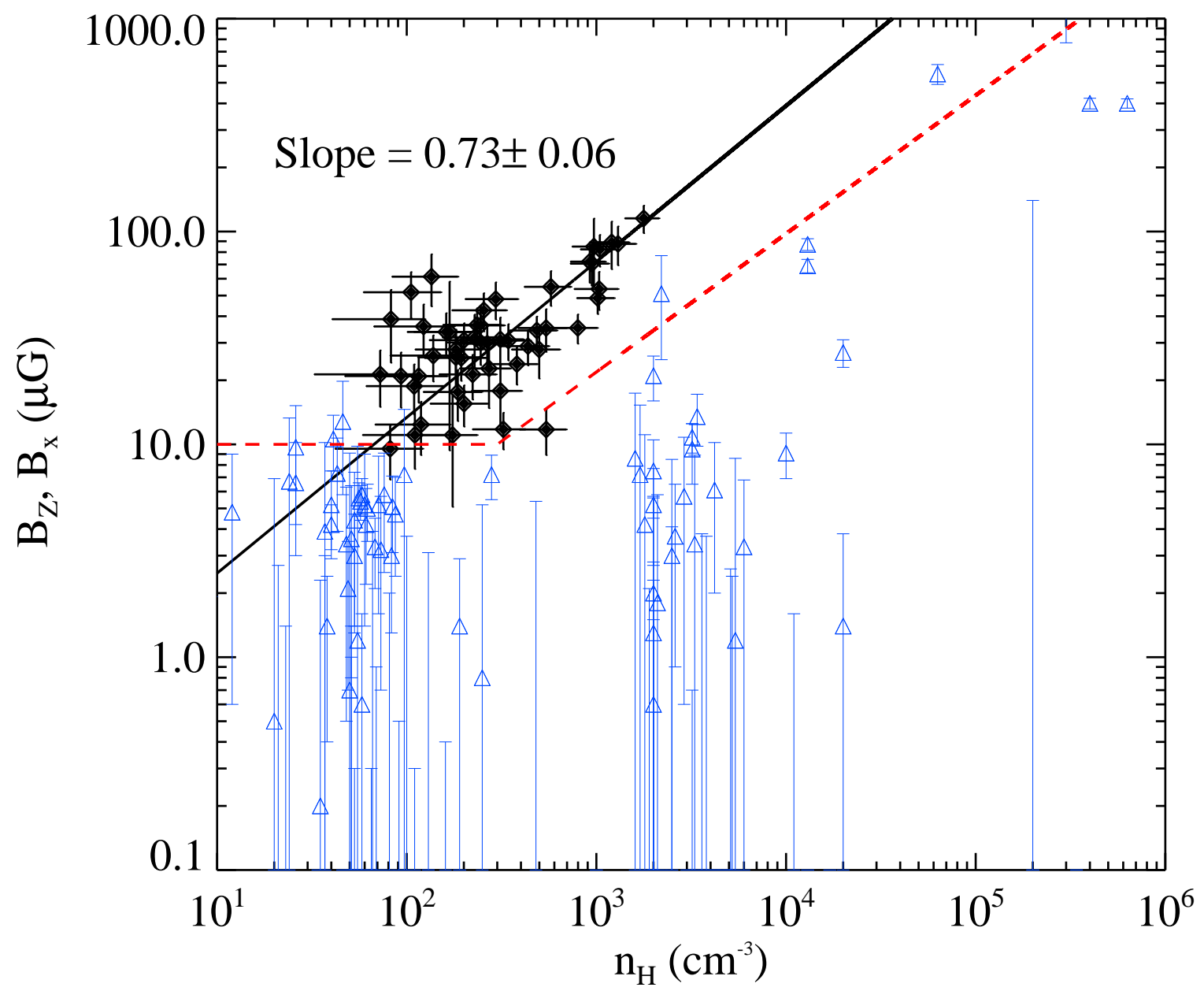

Figure 15. The plane-of-sky B-field strengths of the 51 pixels across G28.23, scaled by $1 / \sqrt{2}$ to become $\mathrm{B}_{x}$, plotted against atomic hydrogen volume density, as the black diamonds. $\mathrm{B}_{z}$ estimates and upper limits from Crutcher et al. (2010) are shown as the blue triangles with corresponding uncertainties. The Crutcher et al. (2010) maximum B-field estimate, $\mathrm{B}_{\max }$, is overlaid as the red, broken, dashed line. A single power law fit to the $\mathrm{B}_{x}$ values of G28.23 is shown as the black solid line, which has been extrapolated to densities outside those probed toward G28.23.

cloud major axis, the average $\mathrm{M} / \Phi_{B N}$ over all possible inclination angles (with respect to the line of sight) will be (Crutcher et al. 2004; Planck Collaboration et al. 2016b):

$$
\overline{M / \Phi}=\int_{0}^{\pi / 2} \frac{M_{\|} \cos \theta}{\Phi_{\perp} / \sin \theta} \sin \theta d \theta=f\left(M_{\|} / \Phi_{\perp}\right)
$$

where $\theta$ is the inclination of the cloud with respect to the line of sight. Limiting geometries include: the Bfield being perpendicular to the cloud major axis, and, the B-field being parallel to the cloud major axis. The correction factor, $f$, applied to $\mathrm{M}_{\|} / \Phi_{\perp}$ to yield the average $\mathrm{M} / \Phi_{B N}$, will be bounded by $1 / 3$ (perpendicular) and 3/4 (parallel) (Planck Collaboration et al. 2016b). Therefore, the $\mathrm{M}_{\|} / \Phi_{\perp}$ values presented for G28.23 need correction, depending on geometry, to infer $\mathrm{M} / \Phi_{B N}$. This correction does assume that all inclination angles are likely, which may not be the case.
Figure 16 shows the $\mathrm{M}_{\|} / \Phi_{\perp}$ map of G28.23. The $\mathrm{M}_{\|} / \Phi_{\perp}$ ratios range from $\sim 0.09-7.5$, with a median of $\sim 1.55$. The $\mathrm{M}_{\|} / \Phi_{\perp}$ values appear to increase with increasing cloud density. This increase can be seen in the figure, where the lowest $\mathrm{M}_{\|} / \Phi_{\perp}$ values are at the cloud edge, while the highest values are closer to the cloud center.

Figure 17 plots the $\mathrm{M}_{\|} / \Phi_{\perp}$ values against atomic hydrogen volume density. The horizontal dashed line represents unity for $\mathrm{M}_{\|} / \Phi_{\perp}$. A positive correlation is seen between density and $\mathrm{M}_{\|} / \Phi_{\perp}$, where regions that are less dense tend to have lower $\mathrm{M}_{\|} / \Phi_{\perp}$ values, and regions that are denser tend to have larger $\mathrm{M}_{\|} / \Phi_{\perp}$ values. Applying the geometric correction factor lowers the $\mathrm{M}_{\|} / \Phi_{\perp}$ estimated $\mathrm{M} / \Phi_{B N}$ values and changes the average volume density at which the cloud goes from subcritical (B-field dominated) to supercritical (gravitationally dominated). Similar to the B-field strength, a power law of the form $\mathrm{M}_{\|} / \Phi_{\perp} \sim n^{\alpha}$ was fit to $\mathrm{M}_{\|} / \Phi_{\perp}$ versus $n$. The best- 


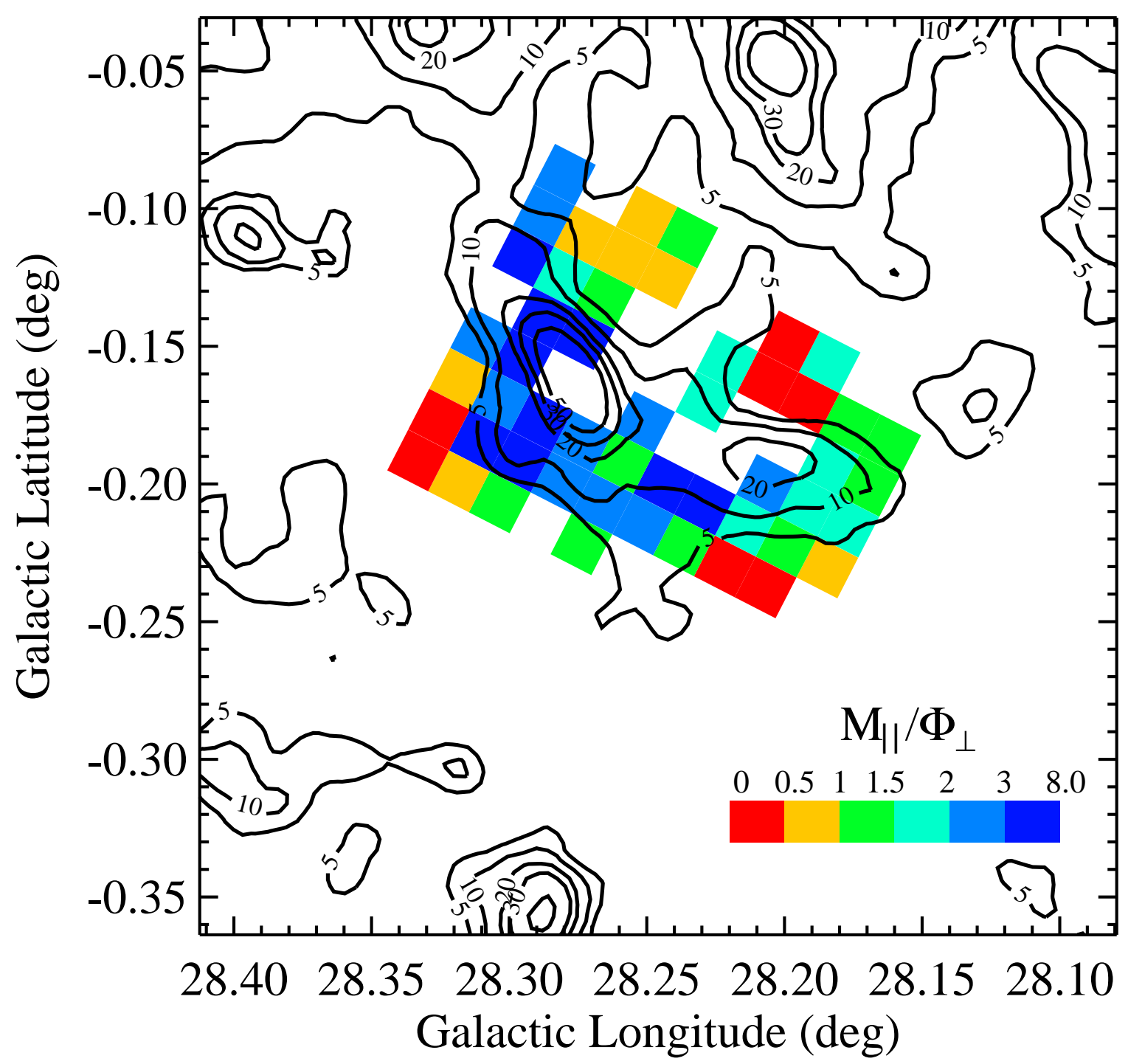

Figure 16. Normalized Mass-to-Flux ratios observed toward G28.23, with values indicated by colors corresponding to the color bar. Column-density-derived $\mathrm{A}_{V}$ contours are overlaid in black.

fit power-law has an index of $1.02 \pm 0.08$. The uncorrected volume density that corresponds to criticality is $225 \mathrm{H} \mathrm{cm}^{-3}$, whereas the corrected critical volume density ranges from $\sim 300$ to $670 \mathrm{H} \mathrm{cm}^{-3}$, depending on the correction factor.

\section{DISCUSSION}

To test the importance of the B-field in IRDC G28.23, we examined the relationships between the B-field and other cloud properties. In this section, we discuss the implications of the results.

\subsection{Plane-of-sky B-field Morphology: Relative PA Orientations}

The first test of the importance of the B-field in the formation of G28.23, Question 2, examines the relative orientation of the polarization PAs with respect to the projected cloud orientation. For this IRDC, the relative PA orientations were neither preferentially perpendicular nor parallel to the cloud major axis orientation. The relative PA orientations do, however, show a large-scale pattern, and appear to have distinct distributions in the Northern and Southern regions of the cloud, as seen in Figure 9, with nearly a $30^{\circ}$ change in median relative PAs across the cloud spine location. The PAs in the North are more likely to be perpendicular to the cloud elongation, especially in the Northeast, whereas in the South (especially Southwest), PAs are more likely to be parallel to the cloud elongation.

Numerical simulations of filament formation in magnetized media (e.g., Van Loo et al. 2014) find that in regions where the $\mathrm{B}$-field is strong, the orientation of the B-field is aligned with that of the cloud. Recent studies of a nearby IRDC, G14.2 (Busquet et al. 2013; Santos 


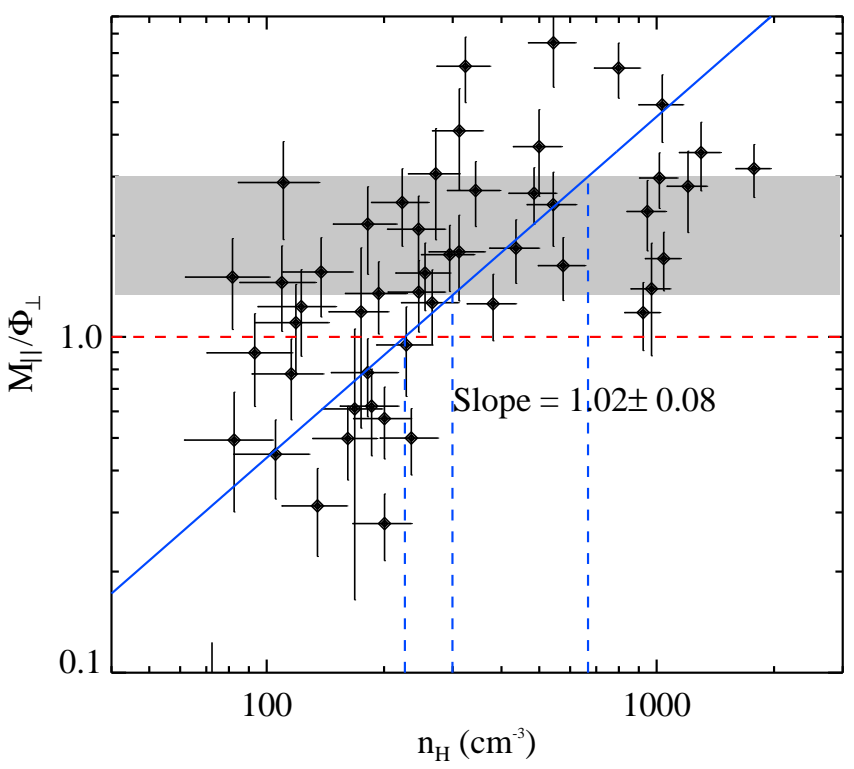

Figure 17. Normalized Mass-to-Flux ratio $\left(\mathrm{M}_{\|} / \Phi_{\perp}\right)$ values plotted against atomic hydrogen volume density, as black diamonds. Similar to Figure 15, a single power law (blue) was fit to the data. The horizontal dashed red line represents unity in uncorrected $\mathrm{M}_{\|} / \Phi_{\perp}$. The gray region represents unity in $\mathrm{M} / \Phi_{B N}$ when $\mathrm{M}_{\|} / \Phi_{\perp}$ is scaled by $1 / 3$ to $3 / 4$, depending on the geometry of the system. The dashed vertical blue lines indicate the corresponding critical densities.

et al. 2016) and the relative orientations between Planck observations and nearby molecular clouds (Planck Collaboration et al. 2016a) have found that the B-field is preferentially perpendicular to dense cloud filaments. Using Herschel observations of a portion of the Taurus Molecular cloud (B211 \& L1495), Palmeirim et al. (2013) found that less dense filaments, which are preferentially parallel to the surrounding B-field, connect perpendicularly to the main filament.

One specific cloud configuration in a strong B-field regime is the hub-filament system, where the B-field helps funnel material to a central dense hub along one or more filaments (Myers 2009; Chapman et al. 2011; Li et al. 2014; Pillai et al. 2015). Such a configuration would exhibit B-fields perpendicular to the dense hub and parallel to the less dense connecting filaments. In simulations of turbulent molecular clouds, Soler et al. (2013) also found that in cloud with high B-field strengths, the relative field orientations changed from parallel to perpendicular as a function of density.

To test this scenario, the relative PA orientations shown in Figure 9 were separated into 'close' and 'far' subsamples by the median distance between the polarization stars and the cloud spine. This median distance was $\sim 3.3 \mathrm{pc}$, and the median distances in the four quadrants ranged from $\sim 2.5$ to 4.1 pc. Figure 18 shows that the relative PAs for the subsample of stars that are located closer to the cloud spine, and thus probe the higher density cloud regions, are more likely to be parallel to the cloud than the PAs for the subsample of stars that are farther from the cloud, and probe the less dense cloud regions. The median angles of the near and far distributions in each quadrant are listed in the first portion of Table 4, as well as the KS probability determining whether the two distributions were drawn from the same parent population.

The relations between the relative PAs and the cloud column density were also found. Figure 19 shows the relative PAs versus the cloud dust emission cloud column density-derived $\mathrm{A}_{V}$ along the line of sight to each star. The PAs shown here are the same ones shown in Figures 9 and 18. The relations were separated into the four cloud quadrants, and the PA variance-weighted best fitting line was found for each one. Similar to the behavior seen in Figure 18, the relative PAs tend to become more parallel to the cloud elongation as a function of increasing column density. This result is in contrast to what would be expected in a hub-filament system, where the nearer PAs would be expected to be perpendicular to the cloud.

This relative PA configuration may be a signature of a helical field (Fiege \& Pudritz 2000). In such a field, the B-field in the outer regions of the cloud would be toroidally dominated and the B-field in the denser regions of the cloud would be poloidally dominated. It is not clear at what density or radius from the cloud spine the transition in the relative orientation is expected. In a study of high-mass filaments, Contreras et al. (2013) found that their density distributions were consistent with that expected of a cloud wrapped by a helical field.

It is evident from images of G28.23, such as Figure 5 , that the cloud spine is not one straight filament, but has some curvature. Therefore, some of the changes in relative PA between the four quadrants could be due to the change in orientation of the filament along the cloud spine. To test for this effect, we separated the Galactic PAs into the four quadrants and near and far distributions, very similar to the relative PAs. If the cloud has affected the orientation of the local B-field, then the GPA distributions should differ between near and far subsamples. The second portion of Table 4 lists the medians and KS probabilities between the near and far GPA distributions for each quadrant. While the near and far distributions of the NE and SE quadrants have similar characteristics, the near and far distributions of the NW and SW quadrants are different. For both quadrants, the near subsamples are more likely to be closer to $90^{\circ}$, or more plane-parallel. 


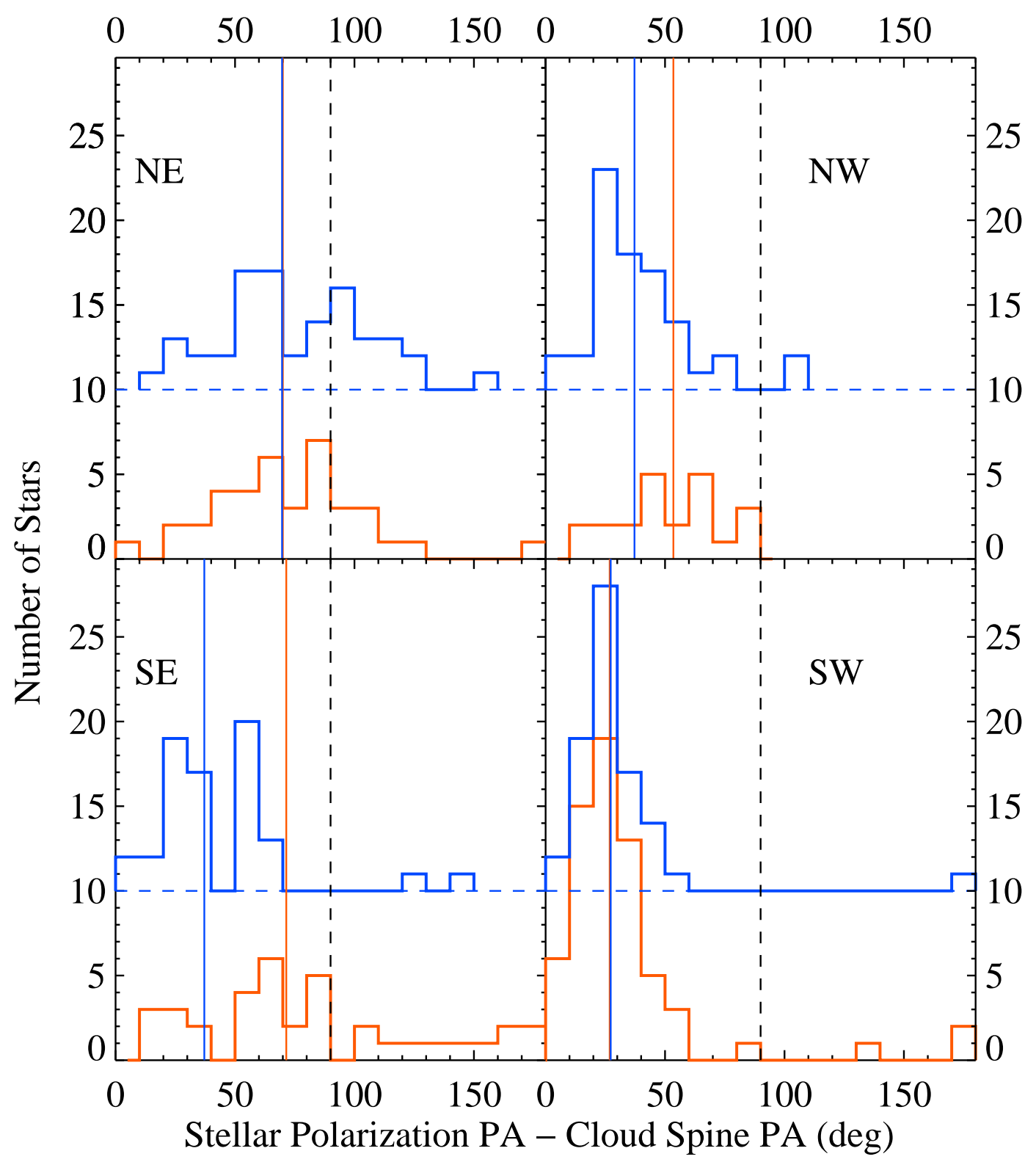

Figure 18. Similar to Figure 9 but relative PA distributions separated into 'near' (blue, offset by 10 counts for clarity) and 'far' (orange) categories based on the average distance between the polarization stars and the cloud spine. The locations of the medians of the distributions are indicated by the vertical color-coded lines. In the Northwest and Southeast quadrants, the PAs closer to the cloud spine are more likely to be parallel to the cloud elongation. 


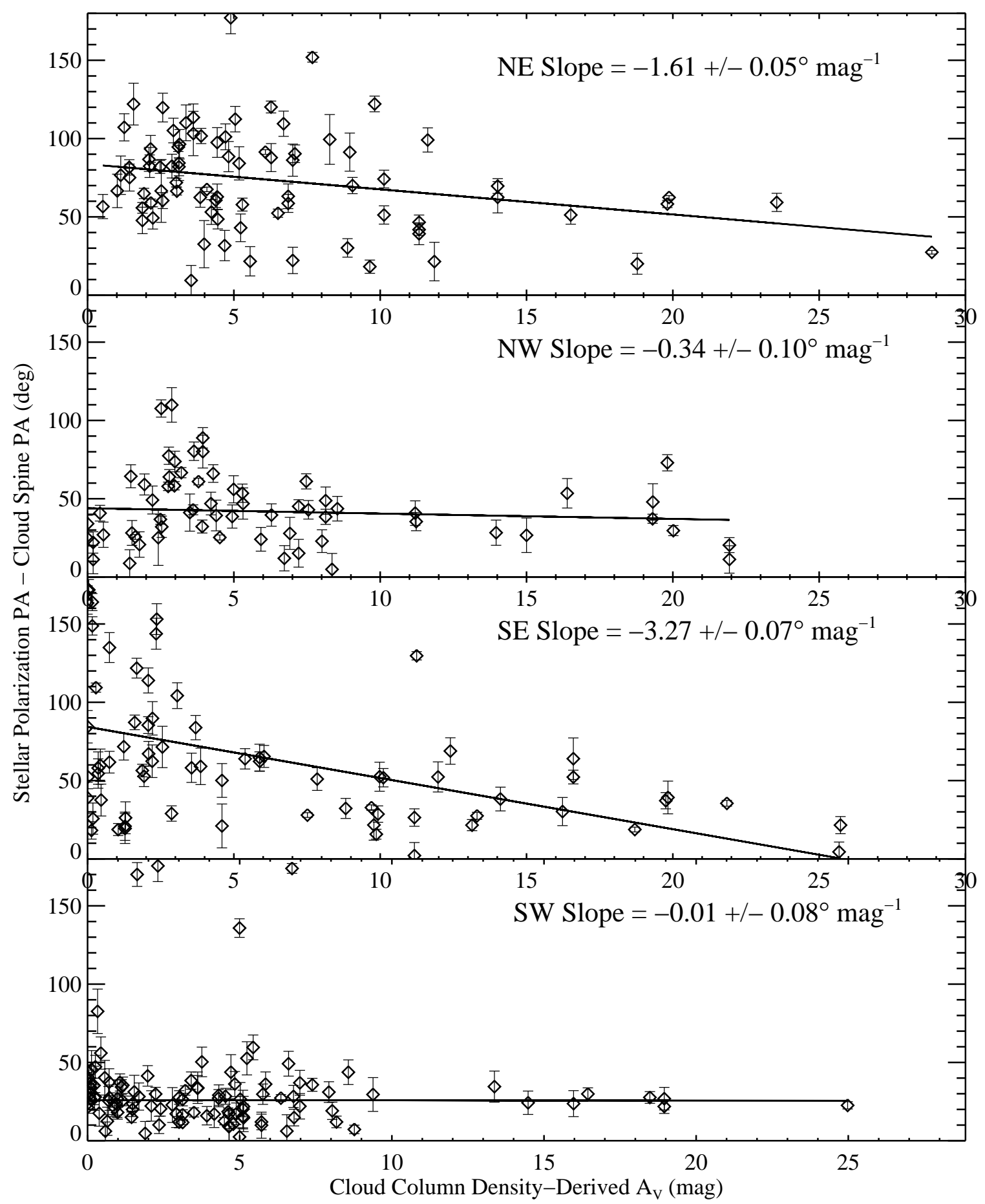

Figure 19. Relative stellar polarization PA versus the cloud column density-derived $\mathrm{A}_{V}$ along the line of sight to each star. The relations have been separated into the two quadrants designated in Figure 8. The relative PAs tend to either remain parallel to the cloud axis (NW, SW) or change from preferentially perpendicular to parallel as a function of cloud column density (NE, $\mathrm{SE})$. 
Table 4. PA Distribution Properties in the four Quadrants

\begin{tabular}{|c|c|c|c|}
\hline $\begin{array}{c}\text { Distribution } \\
\text { Quadrant } \\
(1)\end{array}$ & $\begin{array}{c}\text { Near Median } \\
\text { (deg) } \\
(2) \\
\end{array}$ & $\begin{array}{c}\text { Far Median } \\
\text { (deg) } \\
(3)\end{array}$ & $\begin{array}{c}\text { KS Prob. }{ }^{\mathrm{a}} \\
(4) \\
\end{array}$ \\
\hline$\Delta \mathrm{PA} \mathrm{NE}$ & 69 & 70 & 0.83 \\
\hline$\Delta \mathrm{PA} \mathrm{NW}$ & 37 & 53 & 0.02 \\
\hline$\Delta \mathrm{PA} \mathrm{SE}$ & 37 & 71 & $2 \times 10^{-6}$ \\
\hline$\triangle \mathrm{PA} \mathrm{SW}$ & 27 & 26 & 0.95 \\
\hline GPA NE & 104 & 110 & 0.43 \\
\hline GPA NW & 115 & 131 & 0.03 \\
\hline GPA SE & 107 & 104 & 0.39 \\
\hline GPA SW & 98 & 114 & 0.01 \\
\hline
\end{tabular}

${ }^{a}$ Probability that the near and far distributions are drawn from the same parent population.

Based on the relative PA orientations with respect to locations in the cloud, it is unclear whether the B-field played a dominant role in the formation of G28.23.

We note the presence of a bright $8 \mu \mathrm{m}$ feature in the Southeast region $(l \sim 28.3, b \sim-0.2)$ of the cloud, which may have disrupted the B-field in that area. As seen in Figure 7, the polarization measurements appear to form a shell around this bright ring-like structure. Fewer high SNR stars are present near the structure, compared to the rest of the region. This structure may correspond to a large bubble in the Catalog of bubbles from the Milky Way Project, centered at $l=28.297$ and $b=-0.202$ (Simpson et al. 2012). The bubble may have disrupted either the B-field or the dust grains in the region such that polarizations cannot easily be measured. Spectral information targeting this structure was unavailable at the time of this study. While GRS ${ }^{13} \mathrm{CO}$ line emission at different radial velocities toward this region (lines at 74 and $81 \mathrm{~km} \mathrm{~s}^{-1}$ ) can be detected, this feature is not seen as a coherent structure in the ${ }^{13} \mathrm{CO}$ data. We conclude that the distance to this bubble is not known, and its association with G28.23 remains circumstantial.

\subsubsection{Projection Effects}

One concern in determining the relative angles between polarization measurements and cloud orientations are 3-dimensional projection effects. Both the polarization and cloud orientations are projections onto the 2dimensional plane of the sky of a 3-D B-field and cloud. Hull et al. (2014), using Monte Carlo simulations, explored projection effects in the context of protostellar outflow and B-field orientations. They found that, unless the relative orientations are within 20 degrees of predominantly parallel, projection effects could produce seemingly perpendicular or other orientations.

Projection effects can thus affect the determination of whether the B-field dominated the formation of G28.23 based on the relative projected angles. However, the relative angles between the polarization measurements themselves are unlikely to be affected by projection effects if they measure the same 3-dimensional B-field pointed in one direction. Therefore, the shift in PAs between the Northern and Southern portions of the cloud, where the PAs are more likely to be perpendicular in the North and parallel in the South with respect to the cloud elongation, still indicates that the presence of the cloud changed the shape of the B-field.

\subsection{B-field, Cloud Density, and Mass-to-Flux}

For the regions where $\mathrm{B}_{P O S}$ strengths were estimated for $\mathrm{G} 28.23$, the relation between $\mathrm{B}_{x}$ and volume density was best fit with a single power law of slope $0.73 \pm 0.06$, which addresses Question 3. This slope is approximately equal to the slope of 0.65 found by Crutcher et al. (2010) for B vs. $n$ at densities larger than $300 \mathrm{H} \mathrm{cm}^{-3}$.

Our best-fit slope, which is greater than $2 / 3$, implies that the B-field was not the dominant force in the formation of G28.23. However, the slope of $2 / 3$ corresponds to a temporal evolution of the B-field with density as material undergoes collapse (Li et al. 2015a). The B vs. $n$ relation of G28.23 characterizes instantaneous structure: the present-day dependence of the B-field strength on density across the cloud. If the temporal test of Bfield importance can be applied to the structural relation, then the $\mathrm{B}$ vs. $n$ relation indicates that the $\mathrm{B}$-field did not affect the formation of G28.23 to a large degree. However, the influence of the B-field in the future evolution of the cloud is not known.

Simulations run by Li et al. (2015b) of clump collapse using both initially strong B-fields (Alfvén Mach number $\left.\mathcal{M}_{a} \sim 1\right)$ and weak B-fields $\left(\mathcal{M}_{a} \sim 10\right)$ found similar dependences of the B-field strength on density to those found in this study. Their strong-field simulations exhibited a power law index of 0.7 , which was their timeaveraged value of the index fit to the $\mathrm{B}$ vs. $n$ relation of a 100-cloud sample at times ranging from 0.4 to $0.64 t_{f f}$. This value is very similar to the index of 0.73 found here. In contrast, their power law dependence in the weak-field simulation was 0.57 . The $2 / 3$ dependence of Mestel (1966) applied to weak B-fields, which is not the case for G28.23, but the similar dependence on density is still seen. The Li et al. (2015b) results imply that the $2 / 3$ dependence on density can still arise in a molecular cloud with initially strong B-fields.

The median uncorrected $\mathrm{M}_{\|} / \Phi_{\perp}$ of the cloud is $\sim 1.5$, for which $\mathrm{M} / \Phi_{B N}$ will range from 0.5 to 1.125 when corrected (Question 4). $\mathrm{M}_{\|} / \Phi_{\perp}$ was found to correlate 
with density with a power law index of $\sim 1$. This result indicates that there is a critical density, $n_{\text {crit }}$, above which the cloud is supercritical and no longer B-field supported. The gray region in Figure 17 indicates where $\mathrm{M} / \Phi_{B N}=1$ would be if a geometric correction factor is applied. The best-fit line between density and $\mathrm{M}_{\|} / \Phi_{\perp}$ intercepts this region for densities in the range $\sim 300-670 \mathrm{H} \mathrm{cm}^{-3}$. The lower limit of this range is similar to the threshold density of $300 \mathrm{H} \mathrm{cm}^{-3}$ that Crutcher et al. (2010) associated with a molecular cloud becoming self-gravitating.

The increase in $\mathrm{M}_{\|} / \Phi_{\perp}$ as a function of cloud density implies that the B-field is dynamically important, as this trend is a prediction of ambipolar diffusion (e.g., Ciolek \& Mouschovias 1994). This result contradicts the interpretation that the B-field did not play a dominant role in G28.23's formation based on the relative orientations of the polarization measurements and the power law dependence of the B-field strength on density.

Previous observations of the lifetimes of prestellar cores (e.g., Ward-Thompson et al. 2007), which are estimated to be on the order of a few times the free-fall time, are far shorter than those predicted by ambipolar diffusion models (e.g., Mouschovias \& Ciolek 1999). To account for this discrepancy, it has been suggested that large-scale turbulence, which can create shocks that lead to regions of compressed gas, must be taken into account, in addition to ambipolar diffusion (e.g., Li \& Nakamura 2004; Chen \& Ostriker 2014). In the case of G28.23, we speculate that the B-field may have initially been strong, and that ambipolar diffusion may have occurred in the cloud envelope as mass drifted into the cloud center, but the cloud is now gravitationally dominated in its denser regions.

\subsection{Systematic Uncertainties of the B-field Strength}

The uncertainties reported in Table 3 for the $\mathrm{B}_{P O S}$ strengths are observational uncertainties, propagated from the random uncertainties of the volume density, the PA dispersion, and the ${ }^{13} \mathrm{CO}$ gas velocity dispersion. Systematic uncertainties will also affect the accuracy of the derived properties, and are likely larger than the observational uncertainties (e.g., Crutcher et al. 2004). These systematics rise from assumptions made in calculating the B-field strength. First, because the goal was to calculate the B-field strength in the plane of the sky, ideally, the gas velocity dispersion used in the CF calculation would be the velocity dispersion measured in the plane of the sky from tangential gas velocities. However, as that information is not available, the dispersion along the line of sight was used as the only suitable proxy, though that imposes a velocity isotropy assumption that may not be valid for MHD turbulence.

One parameter we have assumed has no uncertainty is the kinematic distance of $5.1 \mathrm{kpc}$ (Sanhueza et al. 2013). The distance uncertainty affects the volume density uncertainty, which is used in the calculation of the B-field strength. The uncertainty in the distance was estimated as $\sim 10 \%$ (Sanhueza 2015), which contributes $\sim 5 \%$ to the estimated B-field strength.

A remaining unknown is the inclination angle between the orientation of the cloud long axis and the plane of the sky. In the calculation of volume density, we assumed zero inclination. While the true inclination is not known, it is unlikely to be $90^{\circ}$ (the cloud viewed directly down its spine). Another unknown is whether the cloud spine curves with respect to the plane of sky, which would result in multiple inclination angles. For simplicity, the assumption of zero inclination was adopted for the Bfield strength estimate.

The correction factor, f, used in Equation 6, also ranges from 0.46 to 0.51 (Ostriker et al. 2001), which introduces an uncertainty of $\sim 10-15 \%$. The systematic uncertainty of the PA dispersion is dictated by the uncertainty of the measured PAs, which for Mimir is $\sim 0.6^{\circ}$ (Clemens et al. 2012a). This systematic uncertainty is so low that it is usually dominated by the observational uncertainties (a few to $\sim 15 \%$, as listed in Table 3).

One of the largest uncertainties in the B-field calculation comes from the average volume density. Since density is an input variable in the CF B-field strength calculation, the uncertainties of $\mathrm{B}$ and density are not independent. Uncertainties will arise in the B-field strength that depend on the method chosen to estimate gas density. Therefore, the method used to estimate the average volume density affects not only the B-field strength, but also any comparison between the B-field, density, and Mass-to-Flux ratio. The effects of systematic uncertainties of volume density on B-field and $\mathrm{M} / \Phi_{B}$ uncertainties are discussed in detail below. In short, we calculated B-field strengths of G28.23 using a variety of average volume density approaches, and found that the B-field strengths ranged by about a factor of 2 . Therefore, we estimate that our systematic uncertainties for the B-field strengths are about a factor of 2 to 3 .

\subsubsection{Systematic Uncertainties due to Volume Density}

Because the critical density of ${ }^{13} \mathrm{CO}$ (on the order of $10^{3} \mathrm{~cm}^{-3}$ ) is larger than the values found for much of the average volume density map, whether ${ }^{13} \mathrm{CO}$ can become collisionally excited at these densities, and thus be an adequate tracer of the gas motions, becomes an issue. However, ${ }^{13} \mathrm{CO}$ is detected across the entire extent of G28.23. The discrepancy between the critical density of ${ }^{13} \mathrm{CO}$ and the derived average densities in these regions is likely due to beam dilution, where clumpy, localized regions of higher density are averaged with regions of lower density. Mapping this substructure would require 
higher resolution data. Nevertheless, based on how well the $13^{C O}$ integrated intensity traces the dust-based column density, the $13^{C O}$ line widths are likely applicable to calculating the B-field strength in the region.

The average volume density characterizing lines of sight through a cloud can be calculated using different methods (e.g., Marchwinski et al. 2012). These will result in different density estimates. In the present study, the selection of the cloud boundary affects the average volume density estimates. Our chosen boundary of $50 \mathrm{H}_{2} \mathrm{~cm}^{-3}$ might be considered somewhat liberal, whereas selecting a boundary corresponding to the FWHM of the cloud column density may be overly restrictive and would result in ignoring a substantial amount of cloud material and associated B-field.

To determine the effects of the assumptions made in calculating the average volume densities, we recomputed the $\mathrm{B}_{P O S}$ strengths and $\mathrm{M}_{\|} / \Phi_{\perp}$ values using average volume densities derived from other boundary values. Volume density boundaries of 550, 200, and $100 \mathrm{H}_{2} \mathrm{~cm}^{-3}$ corresponded to the cloud column density widths equal to the FWHM, $\pm 2 \sigma$, and $\pm 3 \sigma$, respectively, where $\sigma$ was derived from fitting the cloud column density perpendicular to the cloud spine, as described in Section 3.8. The derived B-field strengths were higher on average for volume densities calculated with stricter cloud boundaries, i.e., for a FWHM boundary. The $\mathrm{B}_{P O S}$ strengths derived using the FWHM boundary ranged from $\sim 10$ $290 \mu \mathrm{G}$. The best-fitting power law indices of $\mathrm{B}_{x}$ vs. $n$ ranged from $1.24 \pm 0.24$ to $0.82 \pm 0.08$ for widths equal to the FWHM and $\pm 3 \sigma$. The best fits to the relations found by using the FWHM boundary are more uncertain because the number of pixels meeting the criteria to calculate B-field strengths decreased drastically (to 15 pixels) compared to the case of the larger boundary out to $50 \mathrm{H}_{2} \mathrm{~cm}^{-3}$ (51 pixels).

The use of different boundaries to calculate the average volume densities for G28.23, while changing the indices of the best-fitting power laws to the $\mathrm{B}$ vs. $n$ relation, does not change the interpretation that the B-field was not likely to be the dominant force in the formation of the cloud. The power law indices of the B vs. $n$ relations are still above the $2 / 3$ threshold, which indicate that gravity was likely the dominant force. This result is still subject to the assumption that the density index for temporal evolution of the B-field matches the density index for present-day structure.

Based on the dependence of the B-field strength on density, the B-field morphology, and the $\mathrm{M}_{\|} / \Phi_{\perp}$ values of the cloud, it is unclear whether the B-field was the dominant force in the formation of G28.23.

\section{SUMMARY}

The importance of the B-field in IRDC formation is not yet understood. To determine whether the B-field played a role in the formation of one IRDC, G28.23, we analyzed archival and new NIR polarimetric observations, along with ancillary archival data. We posed four questions to investigate the role of B-fields in the formation of G28.23. One question addressed whether NIR polarizations could probe the B-field in the intermediate layers of clouds as opaque as IRDCs, and three questions addressed the importance of the B-field in cloud formation. We examined the behavior of NIR polarization percentage with extinction, the relative cloud to B-field PA orientations, the relationship of the B-field strength with density, and finally, the Mass-to-Magnetic Flux ratio across the cloud.

Using a combination of shallow $H$-band and deep $K$ band polarimetric observations, along with dust continuum data from the Herschel Hi-GAL and ATLASGAL surveys, and spectral line data from GRS ${ }^{13} \mathrm{CO}$, we analyzed the properties of the plane-of-sky component of the B-field of G28.23. We found:

1. The polarization percentages increase as a function of extinction, indicating that our observations do probe the B-field of the outer and intermediate layers ( $A_{V}$ values of $30-40 \mathrm{mag}$ ) of G28.23.

2. The overall relative orientation of the plane-of-sky B-field was neither preferentially perpendicular nor parallel to the projected cloud orientation. Therefore, it is unclear from the B-field morphology alone whether the B-field affected the formation of the cloud. The polarization PAs do, however, show a large-scale pattern. The distinct relative PA distributions found in different locations around the cloud indicate that the B-field morphology in the region is affected by the presence of the cloud.

3. The dependence of the B-field strength on cloud density was fit with a power law. The index of the best-fitting power law was $0.73 \pm 0.06$, very similar to the slope of $2 / 3$ that would imply that the B-field was not dynamically important in the formation of the IRDC.

4. The relation between $\mathrm{M}_{\|} / \Phi_{\perp}$ and density, fit with a power law, indicates that the cloud is magnetically dominated at lower densities and becomes gravitationally dominated at higher densities. Applying a correction factor to $\mathrm{M}_{\|} / \Phi_{\perp}$ to account for the unknown geometry of the system shows that the $\mathrm{M} / \Phi_{B N}$ vs. density relation crosses unity in the density range $\sim 300$ $700 \mathrm{H} \mathrm{cm}^{-3}$. The increase in $\mathrm{M}_{\|} / \Phi_{\perp}$ as a function of density implies that the B-field was dynamically important in the cloud's formation, in contrast to the above results.

Based on the B-field properties found by NIR polarimetry in this study, it is unclear whether the B-field influenced the formation of IRDC G28.23. It is likely, 
though, that the local B-field in the vicinity of G28.23 was influenced by the presence and/or formation of the cloud.

\section{ACKNOWLEDGEMENT}

We thank J. Montgomery, T. Hogge, and I. Stephens for constructive discussions on the analysis. We are grateful to R. Crutcher for permission to include his Zeeman data. This research was conducted in part using the Mimir instrument, jointly developed at Boston University and Lowell Observatory and supported by NASA, NSF, and the W.M. Keck Foundation. This research made use of the NASA/IPAC Infrared Science Archive, which is operated by the Jet Propulsion Laboratory, California Institute of Technology (Caltech), under contract with NASA. This publication made use of data products from the Two Micron All Sky Survey, which was a joint project of the University of Massachusetts and the Infrared Processing and Analysis Center/Caltech, funded by NASA and NSF. This work is based in part on data obtained as part of the UKIRT Infrared Deep Sky Survey. The ATLASGAL project is a collaboration between the Max-Planck-Gesellschaft, the European Southern Observatory (ESO) and the Universidad de Chile. It includes projects E-181.C-0885, E-078.F-9040(A), M-079.C-9501(A), M-081.C-9501(A) plus Chilean data. This publication makes use of molecular line data from the Boston University-FCRAO Galactic Ring Survey (GRS). The GRS is a joint project of Boston University and Five College Radio Astronomy Observatory, funded by the National Science Foundation under grants AST-9800334, 0098562, 0100793, 0228993, $\&$ 0507657. A.E.G acknowledges support from FONDECYT 3150570. This work was supported under NSF grants AST 09-07790 and 14-12269 and NASA grant NNX15AE51G to Boston University.

\section{REFERENCES}

Arce, H. G., Goodman, A. A., Bastien, P., Manset, N., \& Sumner, M. 1998, ApJL, 499, L93

Arzoumanian, D., André, P., Didelon, P., et al. 2011, A\&A, 529, L6

Battersby, C., Bally, J., Ginsburg, A., et al. 2011, A\&A, 535, A128

Benjamin, R. A., Churchwell, E., Babler, B. L., et al. 2003, PASP, 115, 953

Bowers, P. F., \& Knapp, G. R. 1989, ApJ, 347, 325

Busquet, G., Zhang, Q., Palau, A., et al. 2013, ApJL, 764, L26

Carey, S. J., Noriega-Crespo, A., Mizuno, D. R., et al. 2009, PASP, 121, 76

Cashman, L. R., \& Clemens, D. P. 2014, ApJ, 793, 126

Chandrasekhar, S., \& Fermi, E. 1953, ApJ, 118, 113

Chapman, N. L., Goldsmith, P. F., Pineda, J. L., et al. 2011, ApJ, 741, 21

Chen, C.-Y., \& Ostriker, E. C. 2014, ApJ, 785, 69

Ciolek, G. E., \& Mouschovias, T. C. 1994, ApJ, 425, 142

Clemens, D. P., Pinnick, A. F., \& Pavel, M. D. 2012a, ApJS, 200, 20

Clemens, D. P., Pinnick, A. F., Pavel, M. D., \& Taylor, B. W. 2012b, ApJS, 200, 19

Clemens, D. P., Sarcia, D., Grabau, A., et al. 2007, PASP, 119, 1385

Contreras, Y., Rathborne, J., \& Garay, G. 2013, MNRAS, 433, 251

Cortes, P. C., Crutcher, R. M., Shepherd, D. S., \& Bronfman, L. 2008, ApJ, 676, 464

Crutcher, R. M. 2012, ARA\&A, 50, 29

Crutcher, R. M., Nutter, D. J., Ward-Thompson, D., \& Kirk, J. M. 2004, ApJ, 600, 279

Crutcher, R. M., Wandelt, B., Heiles, C., Falgarone, E., \& Troland, T. H. 2010, ApJ, 725, 466

Dotson, J. L., Vaillancourt, J. E., Kirby, L., et al. 2010, ApJS, 186,406

Draine, B. T. 2003, ARA\&A, 41, 241

Fiege, J. D., \& Pudritz, R. E. 2000, MNRAS, 311, 85

Girart, J. M., Beltrán, M. T., Zhang, Q., Rao, R., \& Estalella, R. 2009, Science, 324, 1408
Guzmán, A. E., Sanhueza, P., Contreras, Y., et al. 2015, ApJ, 815,130

Heiderman, A., Evans, II, N. J., Allen, L. E., Huard, T., \& Heyer, M. 2010, ApJ, 723, 1019

Heiles, C. 2000, AJ, 119, 923

Hildebrand, R. H., Kirby, L., Dotson, J. L., Houde, M., \& Vaillancourt, J. E. 2009, ApJ, 696, 567

Hennebelle, P. 2013, A\&A, 556, A153

Hull, C. L. H., Plambeck, R. L., Kwon, W., et al. 2014, ApJS, 213,13

Indebetouw, R., Mathis, J. S., Babler, B. L., et al. 2005, ApJ, 619,931

Jackson, J. M., Rathborne, J. M., Shah, R. Y., et al. 2006, ApJS, 163,145

Lada, C. J., Lada, E. A., Clemens, D. P., \& Bally, J. 1994, ApJ, 429,694

Lawrence, A., Warren, S. J., Almaini, O., et al. 2007, MNRAS, 379, 1599

Lazarian, A., \& Hoang, T. 2007, MNRAS, 378, 910

Li, Z.-Y., \& Nakamura, F. 2004, ApJL, 609, L83

Li, H.-b., Dowell, C. D., Goodman, A., Hildebrand, R., \& Novak, G. 2009, ApJ, 704, 891

Li, H.-b., Fang, M., Henning, T., \& Kainulainen, J. 2013, MNRAS, 436, 3707

Li, H.-B., Goodman, A., Sridharan, T. K., et al. 2014, Protostars and Planets VI, 101

Li, H.-B., Yuen, K. H., Otto, F., et al. 2015a, Nature, 520, 518

Li, P. S., McKee, C. F., \& Klein, R. I. 2015b, MNRAS, 452, 2500

Majewski, S. R., Zasowski, G., \& Nidever, D. L. 2011, ApJ, 739, 25

Marchwinski, R. C., Pavel, M. D., \& Clemens, D. P. 2012, ApJ, 755,130

Mestel, L. 1966, MNRAS, 133, 265

Molinari, S., Swinyard, B., Bally, J., et al. 2010, PASP, 122, 314

Mouschovias, T. C. 1979, ApJ, 228, 475

Mouschovias, T. C., \& Ciolek, G. E. 1999, in NATO Advanced Science Institutes (ASI) Series C, Vol. 540, NATO Advanced Science Institutes (ASI) Series C, ed. C. J. Lada \& N. D. Kylafis, 305

Myers, P. C. 2009, ApJ, 700, 1609 
Nakajima, Y., \& Hanawa, T. 1996, ApJ, 467, 321

Nakamura, F., \& Li, Z.-Y. 2008, ApJ, 687, 354

Ormel, C. W., Min, M., Tielens, A. G. G. M., Dominik, C., \& Paszun, D. 2011, A\&A, 532, A43

Ostriker, J. 1964, ApJ, 140, 1056

Ostriker, E. C., Stone, J. M., \& Gammie, C. F. 2001, ApJ, 546, 980

Padoan, P., \& Nordlund, Å. 1999, ApJ, 526, 279

—. 2002, ApJ, 576, 870

Palmeirim, P., André, P., Kirk, J., et al. 2013, A\&A, 550, A38

Pillai, T., Kauffmann, J., Tan, J. C., et al. 2015, ApJ, 799, 74

Planck Collaboration, Adam, R., Ade, P. A. R., et al. 2016a, A\&A, 586, A135

Planck Collaboration, Ade, P. A. R., Aghanim, N., et al. 2016b, A\&A, 586, A138

Rathborne, J. M., Jackson, J. M., \& Simon, R. 2006, ApJ, 641, 389

Sanhueza, P., Jackson, J. M., Foster, J. B., et al. 2013, ApJ, 773, 123

Sanhueza, P. A. 2015, Ph.D. Thesis,

Santos, F. P., Busquet, G., Franco, G. A. P., Girart, J. M., \& Zhang, Q. 2016, ArXiv e-prints, arXiv:1609.08052
Schuller, F., Menten, K. M., Contreras, Y., et al. 2009, A\&A, 504,415

Simpson, R. J., Povich, M. S., Kendrew, S., et al. 2012, MNRAS, 424,2442

Skrutskie, M. F., Cutri, R. M., Stiening, R., et al. 2006, AJ, 131, 1163

Soler, J. D., Hennebelle, P., Martin, P. G., et al. 2013, ApJ, 774, 128

Sridharan, T. K., Rao, R., Qiu, K., et al. 2014, ApJL, 783, L31

Sugitani, K., Nakamura, F., Watanabe, M., et al. 2011, ApJ, 734,63

Tang, Y.-W., Ho, P. T. P., Girart, J. M., et al. 2009, ApJ, 695, 1399

Troland, T. H., \& Crutcher, R. M. 2008, ApJ, 680, 457

Van Loo, S., Keto, E., \& Zhang, Q. 2014, ApJ, 789, 37

Wardle, J. F. C., \& Kronberg, P. P. 1974, ApJ, 194, 249

Ward-Thompson, D., André, P., Crutcher, R., et al. 2007, Protostars and Planets V, 33

Weingartner, J. C., \& Draine, B. T. 2001, ApJ, 548, 296

Whittet, D. C. B., ed. 2003, Dust in the galactic environment

Whittet, D. C. B., Martin, P. G., Hough, J. H., et al. 1992, ApJ, 386,562

Zhang, Q., Qiu, K., Girart, J. M., et al. 2014, ApJ, 792, 116 